\title{
Estado del conocimiento de la fauna de anfibios y reptiles del departamento de Casanare, Colombia
}

\author{
Raúl Pedroza-Banda ${ }^{1,2, *}$, Jhon Jairo Ospina-Sarria ${ }^{1,2}$, Teddy Angarita-Sierra ${ }^{1,2}$, Marvin Anganoy-Criollo ${ }^{1}$, John D. Lynch ${ }^{1}$ \\ ${ }^{1}$ Grupo de investigación en Biogeografía y Cladística Profunda, Laboratorio de Anfibios, \\ Instituto de Ciencias naturales, Universidad Nacional de Colombia, Bogotá, Colombia \\ ${ }^{2}$ Yoluka ONG, Fundación de Investigación en Biodiversidad y Conservación
}

\begin{abstract}
Resumen
Este estudio presenta la revisión actualizada de la riqueza, composición y distribución de los anfibios y reptiles del departamento del Casanare. Se revisaron 1.012 ejemplares depositados en las principales colecciones biológicas del país y se recolectaron 658 ejemplares en cuatro municipios de Casanare. Se obtuvo el registro de 46 especies de anfibios y 65 especies de reptiles, de las cuales, seis corresponden a nuevos registros y dos amplían la distribución conocida en el país. Los municipios de Sácama, Támara y Recetor presentaron los mayores vacíos de información.

Palabras clave: Orinoquía colombiana, herpeto fauna, llanos, piedemonte, distribución.

Current knowledge of the amphibian and reptile fauna in the department of Casanare, Colombia

Abstract

The present study is an updated revision of the richness, composition and distribution of amphibians and reptiles in Casanare. A total of 1,012 specimens from the main Colombian biological collections were examined and 658 specimens were collected in four Casanare municipalities. As a result, we reported 46 species of amphibians and 65 species of reptiles of which six are new records and two extend their known distribution in Colombia. Additionally, we identified Sácama, Támara and Recetor as the municipalities with the poorest knowledge of their amphibians and reptiles.
\end{abstract}

Key words: Orinoquia, herpeto fauna, distribution, savanna, foothill.

\section{Introducción}

Durante la última década, tanto el gobierno nacional, como la empresa privada, las organizaciones no gubernamentales, las universidades y los centros de investigación han volcado su atención sobre los diferentes ecosistemas de Casanare motivados por el acelerado crecimiento económico de la región y las radicales transformaciones de los paisajes naturales de las llanuras y el piedemonte. Esto ha generado un creciente número de investigaciones que han significado avances en el conocimiento de los anfibios y reptiles del departamento.

A pesar de su vasta extensión y de la importancia que ha tomado esta región para el país, en términos biológicos los esfuerzos han sido insuficientes, ya que el desconocimiento de su fauna sigue siendo evidente, sobre todo en lo concerniente a la fauna de anfibios y reptiles, cuya composición se conoce de manera aceptable en la zona del piedemonte llanero, pero no así en la zona de las sabanas o llanuras del Orinoco.

Los primeros registros sobre la herpeto fauna del departamento se deben a Amaral (1931) y Medem $(1965,1981)$ en lo concerniente a los reptiles y a Cochran \& Goin (1970) en cuanto a los anfibios. Tuvieron que pasar tres décadas para que figuraran nuevos registros del departamento, cuando Ruiz, et al., (1996) actualizaron la lista de especies de anfibios del país, con cuyos registros, y los de otras publicaciones, Acosta-Galvis (2000) realizó una nueva revisión de los anfibios de Colombia en la que reportó 22 especies en Casanare. Recientemente, Acosta-Galvis \& Alfaro-Bejarano (2011) actualizaron la información sobre los anfibios de este departamento en los últimos cinco años, pero con base en los registros del piedemonte, los cuales tienen una baja representatividad de los paisajes de sabana, que son los ecosistemas dominantes en Casanare.

Después de los reportes de Amaral (1931) y Medem (1965, 1981), los estudios sobre los reptiles de este departamento escasearon y no fue hasta la década de 1980 cuando se obtuvieron nuevos registros de los saurios de Colombia

\footnotetext{
*Correspondencia:

Raúl Pedroza-Banda,pedrozabanda@gmail.com

Recibido: 4 de julio de 2013

Aceptado: 16 de abril de 2014
} 
reportados por Ayala (1986), quien registró siete especies de lagartijas presentes en Casanare. Posteriormente, Sánchez-C, et al., (1987) presentaron una lista de especies de Colombia en la cual se registraron 52 especies de reptiles asignadas a este departamento. La contribución más reciente al conocimiento de la fauna de reptiles en esta región del país la realizaron Peñuela Recio, et al., (2011) quienes se refirieron a los reptiles de las reservas de la sociedad civil en cuatro municipios de este departamento. Alfaro, et al., (2011) actualizaron la lista de especies de reptiles de Casanare y Cortés \& Sánchez (2011) evaluaron el estado de conservación de los reptiles presentes en el bloque petrolero Cubiro del municipio de San Luis de Palenque.

Hasta el momento, los aportes al conocimiento de la herpeto fauna de Casanare se han centrado en proveer listas de especies, las cuales son una herramienta útil para iniciar cualquier estudio, pero no se ha evaluado la distribución ni se ha intentado detectar sus patrones e identificar las áreas en las que aún hay desconocimiento en el departamento.

Motivados por los problemas a los que se enfrenta la región de la Orinoquía, y con el fin de dar a conocer la diversidad de la herpeto fauna y su distribución en Casanare, en el año 2012 se realizaron exploraciones de campo en busca de anfibios y reptiles en los municipios de Aguazul, Paz de Ariporo, San Luis de Palenque y Trinidad. Se revisaron, así mismo, las principales colecciones de anfibios y reptiles del país en busca de registros de ejemplares de este departamento. Los resultados mostraron un profundo vacío en el conocimiento tanto de la diversidad como de la distribución de la herpeto fauna en este departamento.

\section{Materiales y métodos}

\section{Muestreo}

Se realizaron tres visitas a ocho localidades del departamento de Casanare durante la época seca y el periodo de lluvias. Se visitaron las veredas Cupiagua (5'19'0.12' N, 72³4'59,95" O) y Manoguía $\left(5^{\circ} 10,082^{\prime} \mathrm{N}, 72^{\circ} 38,190^{\prime} \mathrm{O}\right)$ en el municipio de Aguazul, en el piedemonte llanero; Guaracura ( $5^{\circ} 8^{\prime} 40,4^{\prime \prime}$ N, 7058'22,6” O), La Lucha (5²3'15,0” N, 71³7'16,9” O) y La Riverita (5'8'41,2” N, 70 57'27,9” O) en el municipio de San Luis de Palenque; San Vicente $\left(5^{\circ} 18^{\prime} 47,2^{\prime \prime} \mathrm{N}\right.$, $71^{\circ} 22^{\prime} 5,4^{\prime \prime}$ O) y La Cañada (5 $26^{\prime} 18,9^{\prime \prime} \mathrm{N}, 71^{\circ} 36^{\prime} 49^{\prime}$ ' O) en el municipio de Trinidad y la vereda La Colombina $\left(6^{\circ} 2^{\prime} 36,5^{\prime}\right.$ ' N, 71'5'34,2” O) en el municipio de Paz de Ariporo, sobre los llanos orinocenses (Figura 1).

En el trabajo de campo se empleó la técnica de inventario por búsqueda libre sin restricciones (Rueda, et al., 2006), con salidas diurnas y nocturnas de ocho horas diarias durante un periodo de 36 días (31 días en las localidades sobre los llanos orientales y cinco en la localidad del piedemonte).

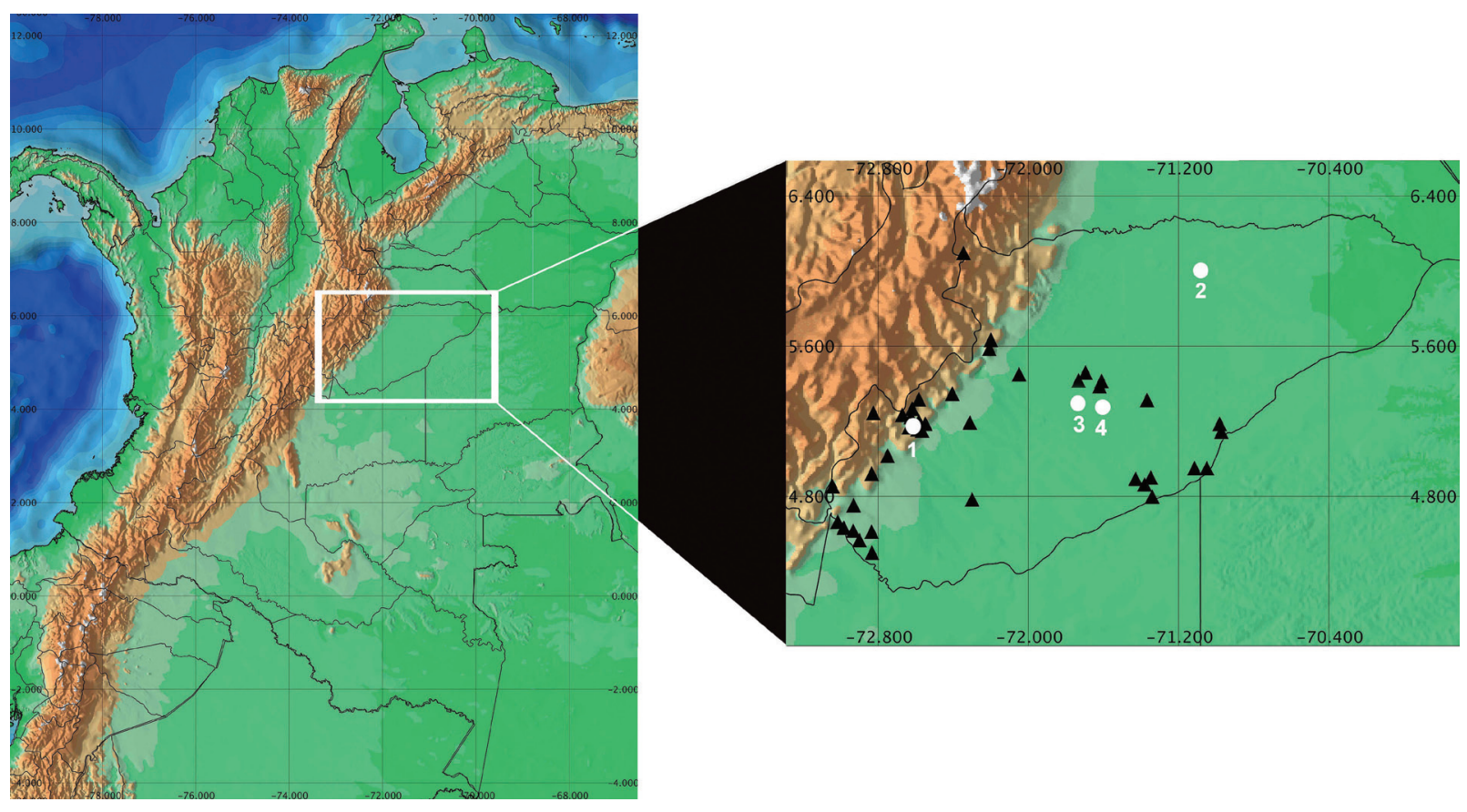

Figura 1. Distribución de las localidades con registros de anfibios y reptiles en el departamento de Casanare, Colombia (Anexo 1). 1. Círculo blanco: localidad visitada en Aguazul (piedemonte); 2. Círculo blanco: localidad visitada en Paz de Ariporo (llanos); 3. Círculo blanco: localidades visitadas en San Luis de Palenque (llanos). 4. Círculo blanco: localidades visitadas en Trinidad. 
Tres investigadores participaron en los muestreos de las localidades en los municipios de Aguazul y Paz de Ariporo, en tanto que en las localidades del municipio de San Luis de Palenque participaron dos investigadores. Se capturaron adultos y renacuajos. Los individuos adultos se almacenaron en etanol $(70 \%)$ y los renacuajos, en formaldehido $(10 \%)$, y se depositaron en la colección de anfibios del Instituto de Ciencias Naturales (ICN) de la Universidad Nacional de Colombia, sede Bogotá.

\section{Revisión de colecciones}

Se visitaron las colecciones del Museo de La Salle, Bogotá, del Museo de Historia Natural de la Pontificia Universidad Javeriana, Bogotá, del Museo de Historia Natural ANDES, Bogotá, del Instituto Alexander von Humboldt y del Instituto de Ciencias Naturales de la Universidad Nacional de Colombia para la búsqueda y revisión de ejemplares de anfibios y reptiles del departamento de Casanare. Esta revisión incluyó tanto ejemplares catalogados como aquellos en proceso de inclusión en las colecciones, para lo cual los datos de las localidades se obtuvieron directamente de las notas de campo.

\section{Determinación taxonómica}

La determinación de las especies se realizó con base en revisiones y claves taxonómicas, ejemplares tipo depositados en el Instituto de Ciencias Naturales de la Universidad Nacional, ejemplares de referencia, descripciones originales de las especies y ayuda de los expertos M. Harvey (teiidos) y R. Caicedo Portilla (gekkonidos).

Las taxonomías empleadas en el presente artículo fueron las de Grant, et al., (2006) para Aromobatidae, la de Faivovich, et al., (2005) para Hylidae, la de Hedges, et al., (2008) para Strabomantidae y la de Frost, et al., (2006) para el resto de familias. En el caso de los reptiles, se siguió a Wüster (2001), a Lehr (2002) y a Campbell y Lamar (2004), en cuanto a las serpientes, a Pyron, et al., (2011), a Harvey (2012), a Caicedo-Portilla \&Dulcey-Cala (2011), y a Poe (2013) para las lagartijas del género Anolis. En el caso de las tortugas y cocodrilos se siguió a Rueda-Almonacid (2007).

\section{Análisis de datos}

Fase de campo. Para los registros obtenidos en campo, la diversidad alfa o riqueza de especies se definió como el número de especies en el área de estudio; la riqueza esperada se calculó por medio del estimador no paramétrico Jacknife de primer y segundo orden. Con el valor máximo de riqueza estimado, se determinó la representatividad del estudio en términos de porcentaje. Los estimadores se calcularon con el programa EstimateS 8.2.0 (Colwell, 2006). Este análisis no se realizó para el ensamble de reptiles, puesto que los resultados con los estimadores clásicos arrojaron resultados no confiables.
Conscientes de que la interpretación de los estimadores de diversidad comúnmente utilizados (Shannon, Simpson) es bastante compleja porque estos incorporan tanto la riqueza de especies como la equidad en un único valor (Ludwing y Reynolds, 1988), es decir, el mismo valor de diversidad puede obtenerse para una comunidad con baja riqueza y alta equidad como para una con alta riqueza y baja equidad, se utilizaron los números de Hill como estimadores debido a la facilidad para interpretarlos, ya que sus resultados se dan en unidades que corresponden al número de especies. Estos números permiten una aproximación a la estructura de las comunidades en el área de estudio (especies en relación con su abundancia) y garantizan que los resultados puedan compararse en el tiempo. La serie de números de Hill considerada fue la siguiente:

N_0: número total de especies (S)

N_1: número de especies abundantes $=\mathrm{e} \mathrm{H}$ ', y

N_2: número de especies muy abundantes $=1 / \lambda$,

donde H' es el índice de Shannon-Wiener y $\lambda$ corresponde al índice de Simpson. Los resultados se dan en unidades de número de especies. Como se puede observar, N_1 y N_2 dan la estimación del número de especies abundantes y muy abundantes por cada una de las unidades de muestreo.

\section{Análisis de distribución de anfibios}

Con base en la información obtenida en las salidas de campo y la recopilada de los registros en las colecciones biológicas visitadas, se buscó identificar patrones de distribución que permitieran delimitar las zonas del piedemonte y de los llanos orientales en las que tradicionalmente se ha subdividido el departamento de Casanare. Para este análisis se partió de la lista general de especies de anfibios del departamento, excluyendo los registros de especies con poblaciones transandinas, es decir, especies con distribuciones al occidente de los Andes. De esta manera se obtuvo una lista final de especies con distribución restringida al oriente de los Andes que fue empleada para determinar si existía o no un patrón de distribución.

\section{Resultados}

\section{Fase de campo}

Se registraron 658 individuos adultos y cuatro lotes de renacuajos y se registraron posturas y cantos del centrolénido Hyalinobatrachium munozorum después de un esfuerzo de muestreo de 640 horas/hombre. Estos registros correspondieron a 35 especies de anfibios (anexo 1) del orden Anura y 28 especies de reptiles (anexo 2).

Las familias de anfibios más representativas fueron la Hylidae, con quince especies y Leptodactylidae con once especies; de la familia Bufonidae se registraron cuatro 
especies y de Strabomantidae y Centrolenidae, dos especies en cada una. En las familias restantes (Microhylidae y Ranidae) se registró una especie en cada una (Figura 2).

En el caso de los reptiles, las familias más diversas fueron la Colubridae, con doce especies, Teiidae, con tres especies, y Amphisbaenidae, Gekkonidae y Sphaerodactylidae, con dos especies cada una. De las familias restantes (Boidae, Testudinidae, Dactyloidea, Gymnophtalmidae, Iguanidae, Podocnemididae y Viperidae) se registró una especie en cada una (Figura 3).

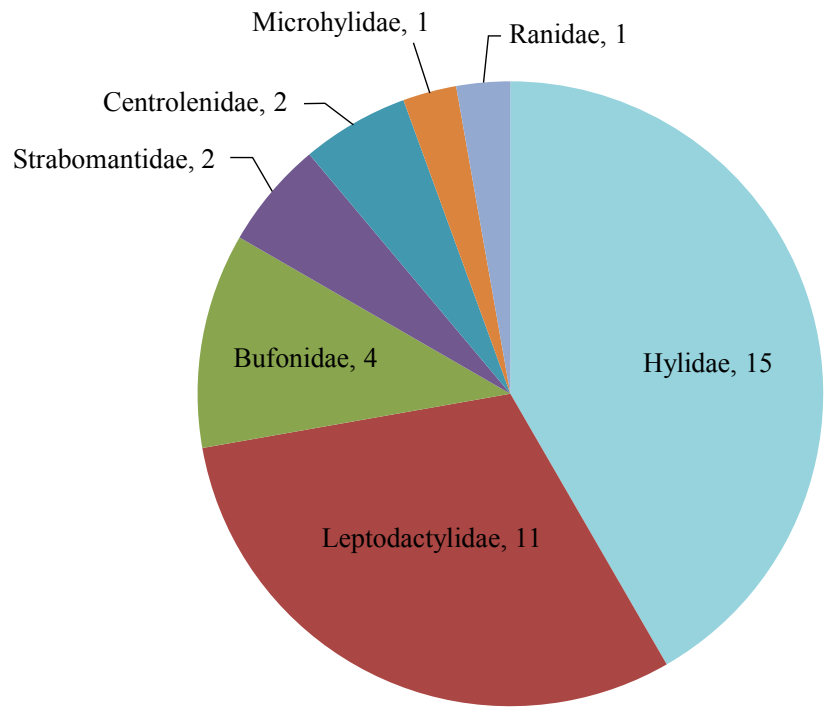

Figura 2. Número de especies de anfibios por familia registradas en la fase de campo

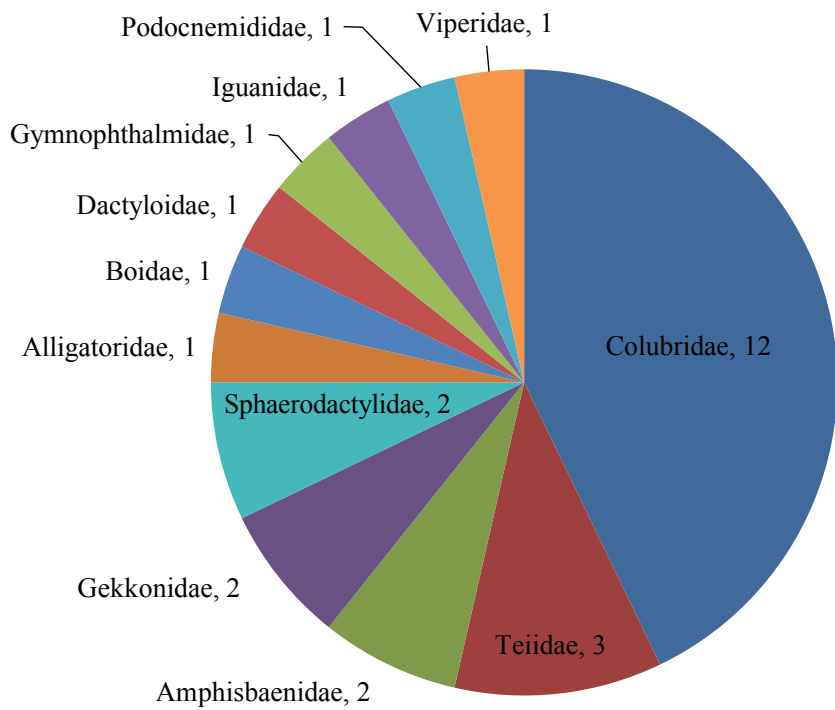

Figura 3. Número de especies de reptiles por familia registradas en la fase de campo

\section{Representatividad del muestreo}

La ausencia de tendencia a alcanzar la asíntota de la curva de acumulación de especies de anfibios en el municipio de Aguazul indica que el muestreo no fue suficiente para acercarse a la representatividad total de especies en el área. Por esto, el número de 14 especies registradas en la localidad de este municipio estuvo por debajo del número estimado de especies esperado (Figura 4a). La curva de acumulación de especies de anfibios en las localidades de los llanos orientales indica que el muestreo fue insuficiente para lograr la representatividad de especies esperada para el ensamble de anfibios de estas localidades (Figura 4b).

Los estimadores empleados indican que el número posible de especies de anfibios para las localidades del municipio de Aguazul estaba en el rango de 19 a 20 especies, por lo cual las especies observadas corresponden a un 74,5\% de representatividad según el estimador Jacknife de primer orden y a 69,5\% según el estimador Jacknife de segundo orden. Los estimadores también indicaron un mejor desempeño en la muestra de las localidades de los llanos orientales, donde se esperaba encontrar entre 29 y 31 especies de anfibios. Por lo tanto, se estima que se logró una representatividad entre 86,6\% (Jacknife de primer orden) y $81,2 \%$ (Jacknife de segundo orden) de la fauna anfibia en esta zona.

\section{Estructura de los ensambles de anfibios}

La abundancia proporcional obtenida para cada una de las localidades visitadas durante los muestreos demostró que en el municipio de San Luis de Palenque y Aguazul se encontraron siete especies abundantes, mientras que en Paz de Ariporo se encontraron seis especies abundantes (Figura 5). Al comparar la identidad de estas especies consideradas abundantes, se demostró que las siete especies registradas en Aguazul (Pristimantis medemi, Rhaebo glaberrimus, Rhinella margaritifera, Leptodactylus colombiensis, Hypsiboaspuntactus, Hypsiboas boanse Hypsiboas lanciformis) eran diferentes a las especies consideradas como abundantes en San Luis de Palenque (Leptodactylus fragilis, Pseudopaludicola llanera, Leptodactylus macrosternum, Leptodactylus fuscus, Hypsiboas crepitans, Dendropsophus mathiassoni y Rhinella humboldti) y en Paz de Ariporo (P. llanera, L. fuscus, Trachycephalus typhonius, Engystomops pustulosus, H. crepitans y Scinax wandae), mientras que estos dos últimos municipios compartían tres especies abundantes (P. llanera, L. fuscus, H. crepitans). Por otro lado, cada una de las localidades muestreadas presentó una especie muy abundante (Figura 5), única para cada una de ellas; en el caso del municipio de Aguazul, la especie muy abundante fue $P$. medemi, en San Luis de Palenque fue L. fragilis y en el municipio de Paz de Ariporo, P. llanera. 
$\mathbf{A}$
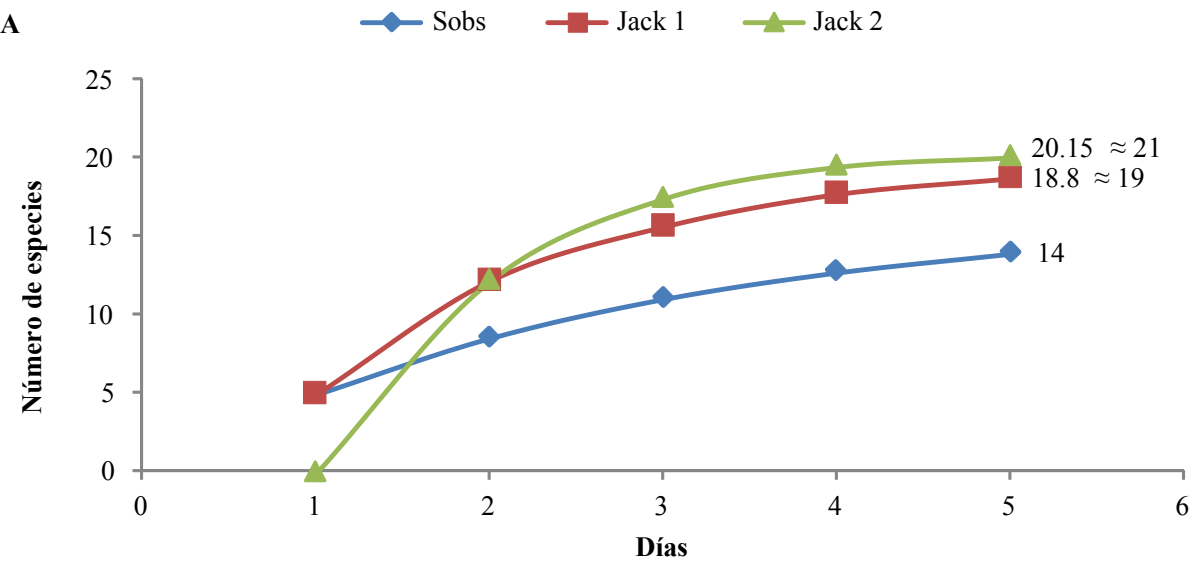

B
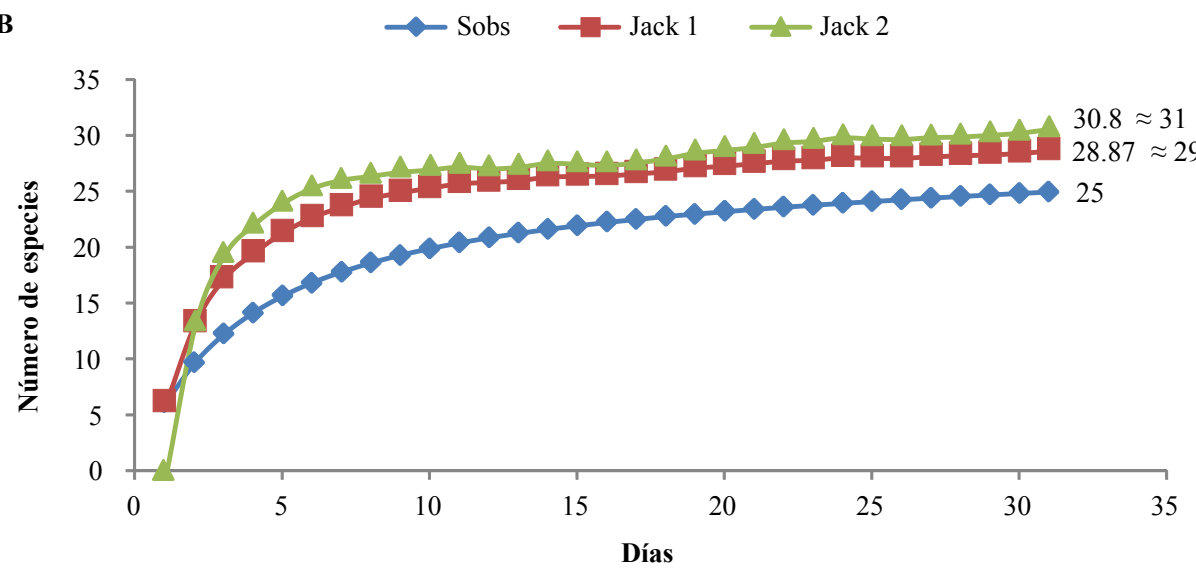

Figura 4. Curva de acumulación de especies para las especies de anfibios registradas en las localidades de (A) piedemonte y (B) llanos.

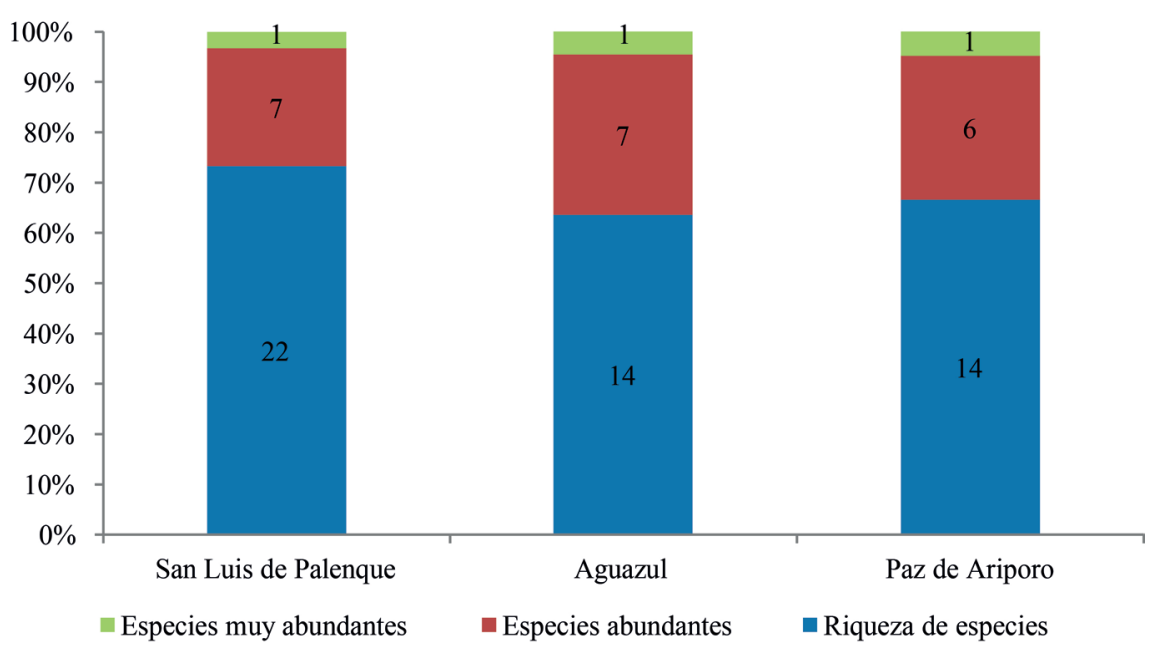

Figura 5. Serie de Hill obtenida para cada una de las localidades visitadas (Aguazul, Paz de Ariporo y San Luis de Palenque) 


\section{Registros de anfibios y reptiles de Casanare en colecciones biológicas}

En total se examinaron 1.012 ejemplares de anfibios y reptiles depositados en diferentes colecciones, de los cuales 38 especies correspondieron a anfibios y 44, a reptiles (Anexos 1 y 2). Estos datos, más los obtenidos en la salida de campo, arrojaron un total de 46 especies de anfibios y 58 especies de reptiles. Además, se espera corroborar la presencia de seis especies más de reptiles (Chelonoidis carbonaria, Chelus fimbriatus, Crocodylus intermedius, Mabuya altamazonica, Paleosuchus trigonatus y Siphlophis compresus), ya que muchos de los registros no estaban disponibles para su revisión durante las consultas realizadas en las colecciones. Sin embargo, se cuenta con los registros fotográficos y la constancia de depósito por parte de los investigadores en las correspondientes colecciones biológicas.

\section{Diversidad de anfibios y reptiles de Casanare}

Entre las 46 especies de anfibios, las familias con mayor representatividad de especies fueron Hylidae, con 20 especies, y Leptodactylidae, con 13. De la familia Bufonidae se encontraron cuatro especies, mientras que de las familias Aromobatidae, Centrolenidae y Strabomantidae se se encontraron dos especies. De las familias restantes (Caeciliidae, Microhylidae yRanidae), se encontró una especie de cada una (Figura 6). Hasta el momento no se tienen registros de especies del orden Caudata (salamandras) en el departamento.

En cuanto a los reptiles, el número total de especies en el departamento de Casanare corresponde a 65 especies, de las cuales, 39 son serpientes y, de estas, 27 pertenecen a la familia Colubridae, cuatro a Boidae, tres a Elapidae, dos a Viperidae, dos a Leptotyphlopidae y una a familia Anomalepididae. Se encontraron cuatro especies de lagartijas de la familia Teiidae, mientras que de las familias Alligatoridae, Amphisbaenidae, Gekkonidae y Dactyloidea se encontraron tres especies de cada una. Se encontraron dos especies en cada una de las familias Sphaerodactylidae y Gymnophthalmidae y de las familias restantes (Chelidae, Iguanidae, Polychrotidae, Scincidae y Testudinidae) se encontró una especie de cada una (Figura 7). Además, se obtuvieron tres nuevos registros de serpientes (Dipsas pavonia, Micrurus filifomis, Thamnodynastes

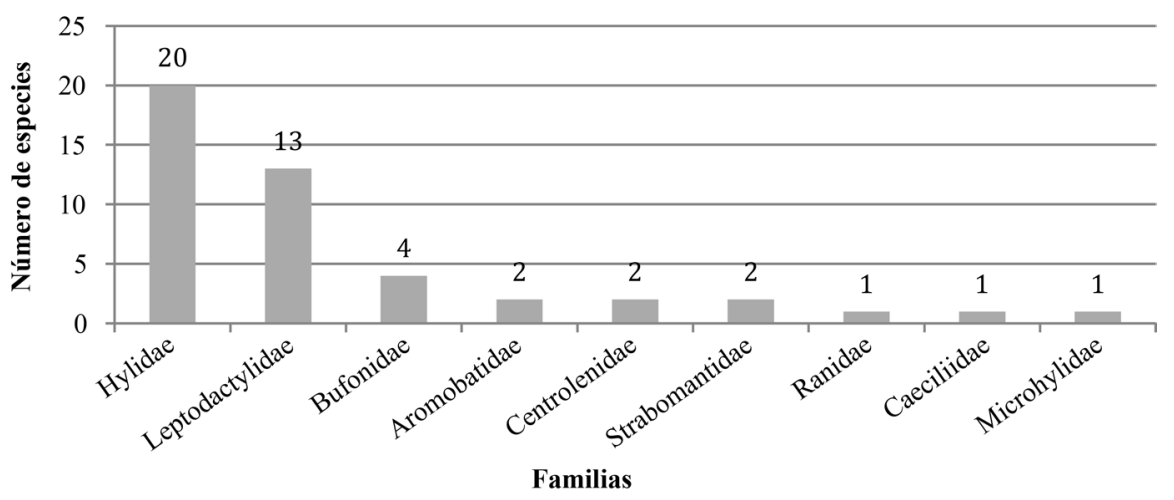

Figura 6. Número total de especies de anfibios por familia registradas para el departamento de Casanare, Colombia

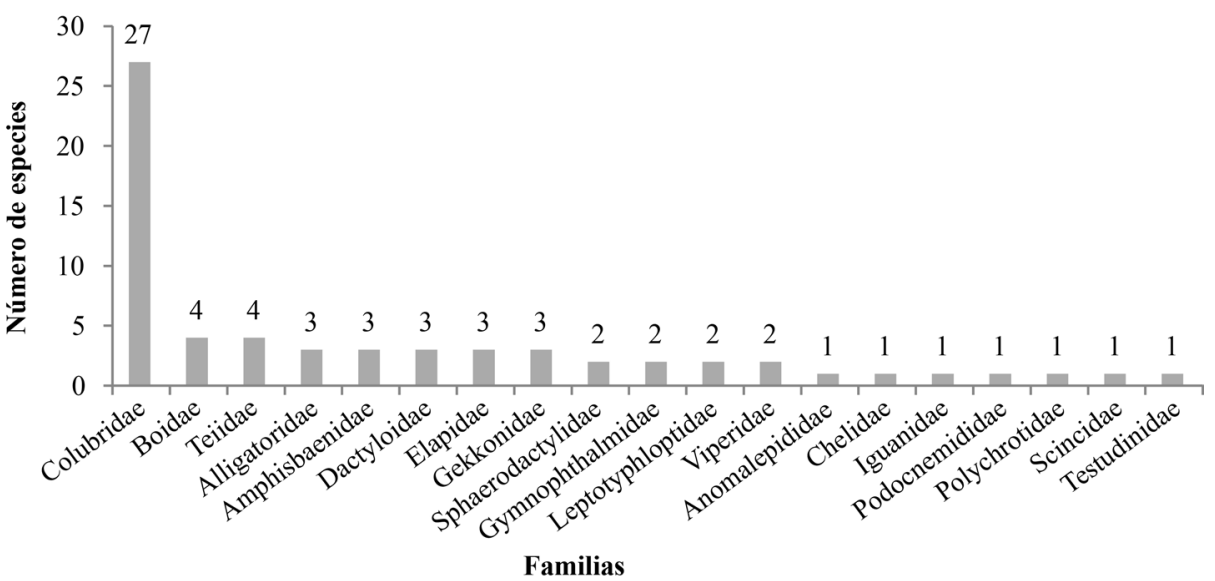

Figura 7. Número total de especies de reptiles por familia registradas para el departamento de Casanare 
dixoni), un nuevo registro de salamanquejas (Hemidactylus palaichthus) y posiblemente dos nuevas especies en el departamento: una serpiente del género Drymarchon y una anfisbena del género Amphisbaena.

\section{Representatividad de los registros de la herpeto fauna de Casanare por municipio}

El departamento de Casanare tiene 19 municipios, en tres de los cuales no hay ningún registro de anfibios o de reptiles (Sácama, Támara y Recetor). En los municipios de Chámeza, Maní y Monterrey no se tienen registros de anfibios, mientras que en Hato Corozal, Pore, Sabanalarga y Villanueva no se tienen registros de reptiles. Los municipios con mejor representatividad son Aguazul, San Luis de Palenque, Trinidad y Paz de Ariporo (Figura 8).

\section{Distribución geográfica de las especies de anfibios en Casanare}

De las especies de anfibios registradas para el departamento de Casanare, solo 32 tienen poblaciones cisandinas. Estas especies se pueden agrupar en tres grupos (Anexo 1): el primero (A), compuesto por 17 especies comunes en piedemonte; el segundo (B), compuesto por once especies comunes en áreas abiertas como los llanos en temporada seca, de lluvias o en ambas, y el tercero (C), compuesto por cuatro especies que habitan comúnmente en los bosques de galería de los Llanos Orientales.

\section{Discusión}

\section{Diversidad de anfibios y reptiles de Casanare}

Con los resultados obtenidos durante la salida de campo en los municipios de Aguazul, Paz de Ariporo y San Luis de Palenque, se lograron seis nuevos registros de anfibios (Hyalinobatrachium munozorum, Leptodactylus sp., Leptodactylus sp.1, Osteocephalus carri, Rulyrana flavopunctata y Scinax sp.). Los registros de Hypsiboas geographicus y Osteocephalus carri son los reportados más al norte de Colombia, pues Cochran \& Goin (1970) reportaron la primera de estas especies en la localidad de Guaicaramo al sur de Casanare, en límites con el departamento de Meta, y Lynch (2006) reportó la segunda especie en el municipio de Restrepo, Meta. Es igualmente importante resaltar el registro de Hypsiboas geographicus, pues esta especie es común en los bosques de la Amazonía. En el caso de los reptiles, se encontraron nuevos registros de seis especies en el departamento (Ameiva preasignis, Amphisbaena fuliginosa, Amphisbaena sp., Drymarchonsp., Hemidactylus palaichtus y Lepidoblepharis sp.).

Los bajos porcentajes de representatividad obtenidos en los muestreos hechos en las localidades del piedemonte, comparados con los de las localidades de los llanos, eran de esperarse debido al corto tiempo disponible para los muestreos en esas localidades, lo cual ocasionó que muchos hábitats y

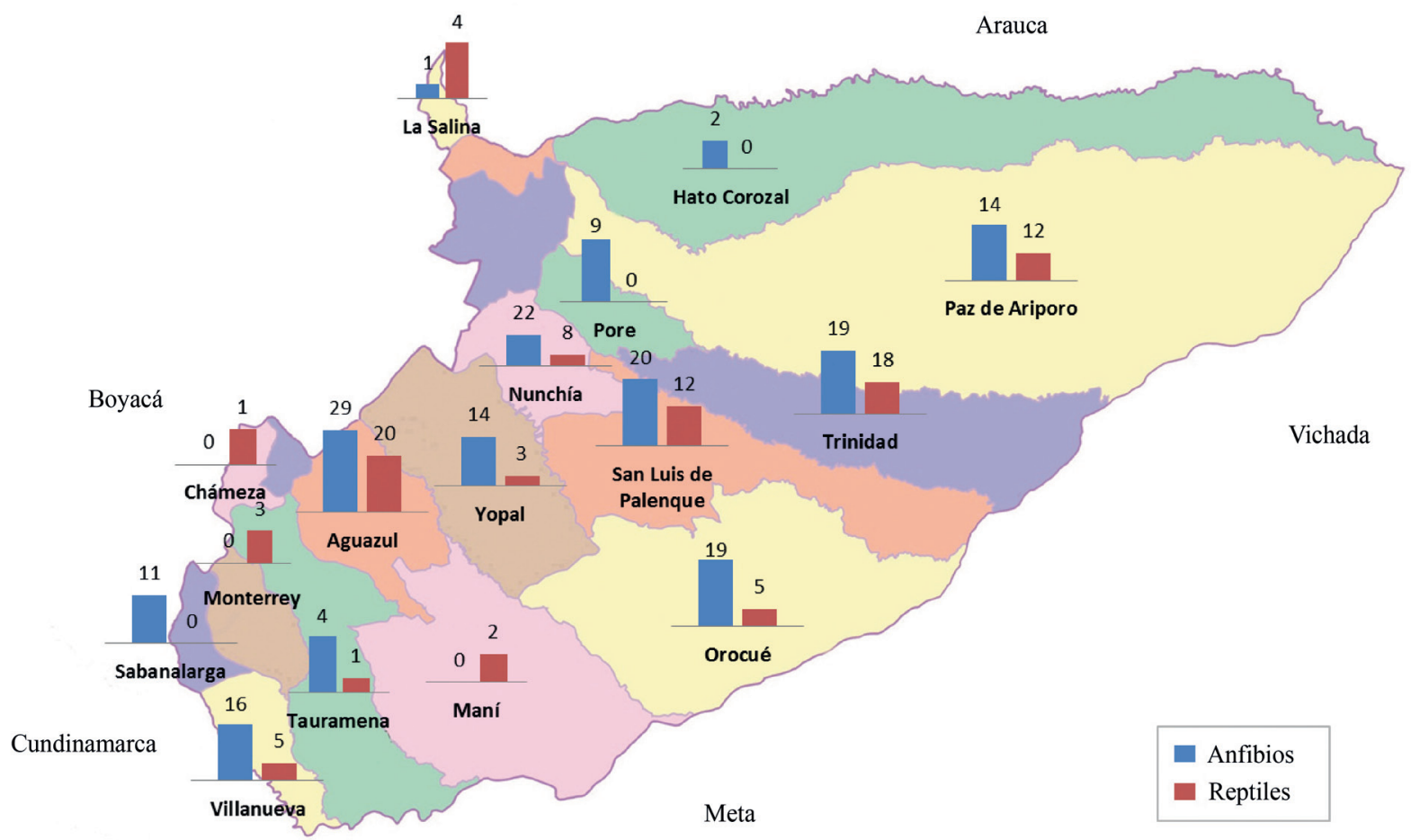

Figura 8. Mapa del departamento de Casanare en donde se muestra el número de especies de anfibios y reptiles registradas por cada municipio (adaptado de Sistema de Información Geográfica Agustín Codazi, 2012) 
microhábitats quedaran excluidos de las búsquedas o fueran muestreados parcialmente. Los hallazgos de especies no registradas antes en Casanare (R. flavopunctata y O. carri), en una zona tan estudiada como lo es el piedemonte, hace suponer que se subestimó el número de especies de anfibios esperado (20 a 19) según el cálculo con los estimadores seleccionados.

El hecho de que ninguna de las especies de anfibios registrada como abundante en la localidad de Aguazul haya sido encontrada en las otras dos localidades no constituye una sorpresa, ya que las condiciones ecológicas de esta localidad son bastante diferentes a las de las otras dos. Aguazul está compuesta por bosques, lo que brinda condiciones de humedad y temperatura distintas a las de las otras dos localidades. Por otra parte, la coincidencia de especies en las localidades de Paz de Ariporo y San Luis de Palenque era de esperarse. En estas dos localidades la cobertura natural predominante es la de sabana, preferida especialmente por $P$. llanera y L. fuscus, cuyo modo reproductivo está adaptado a ambientes adversos para los anfibios (Heyer, 1969), como los que ofrece la sabana natural.

\section{Representatividad de la herpetofauna de Casanare a nivel nacional y local}

Los resultados obtenidos en el trabajo de campo son complementarios de los registros obtenidos en las colecciones científicas visitadas. Al comparar el grado de representatividad de las especies encontradas en el departamento de Casanare respecto a lo registrado en toda la cuenca del Orinoco por Acosta-Galvis, et al., (2010), se encontró que Casanare alberga aproximadamente el 18\% de todas las especies de anfibios registradas hasta el momento, y $22 \%$ de los reptiles.

Los resultados aquí obtenidos concuerdan en un 75,5\% con la riqueza de anfibios reportada por Acosta-Galvis y AlfaroBejarano (2011) y en un 63,1\% con la riqueza de reptiles reportada por Alfaro, et al., (2011) para el departamento. En estos trabajos hay algunos registros dudosos como el de Hypsiboaspugnax (Acosta-Galvis y Alfaro-Bejarano, 2011) y las serpientes Thamnodynastes pallidus y Bothrops asper (Alfaro, et al., 2011). Estos registros no pudieron revisarse debido a que en los estudios citados no se reportaron las colecciones en las cuales residen los ejemplares para así comprobar su identidad taxonómica. Por este mismo motivo, el $24,5 \%$ de los anfibios reportados por Acosta-Galvis y Alfaro-Bejarano (2011) y el 36,9\% de los reptiles reportados por Alfaro, et al., (2011) no se incluyeron en este estudio.

A diferencia de lo que ha ocurrido en el piedemonte, en donde se han centrado los estudios herpetológicos en Casanare y en toda la cuenca del Orinoco, como lo han señalado AcostaGalvis, et al., (2010), el desconocimiento generalizado de las llanuras de la Orinoquía y, específicamente de zonas como el sur de los municipios de Yopal y Tauramena, el occidente de los municipios de Orocué y San Luis de Palenque (mejor conocido en su parte oriental), el norte del municipio de La Salina y prácticamente toda la extensión del municipio de Paz de Ariporo, dificulta la caracterización de la fauna anfibia y de reptiles de las sabanas naturales de Casanare.

La falta de estudios en estas áreas se deben, fundamentalmente, al desarrollo económico, a intereses taxonómicos y biológicos particulares de grupos de trabajo y a la accesibilidad a las diferentes subregiones de la cuenca del Orinoco (Acosta-Galvis, et al., 2010). En el caso de Casanare, esto se debe, principalmente, a la dificultad del acceso a las áreas no exploradas del oriente de los municipios de Hato Corozal y Paz de Ariporo.

\section{Análisis de la distribución de los anfibios de Casanare}

El único patrón de distribución detectado corresponde a aquellos anfibios restringidos en los bosques de piedemonte (grupo A) y esto es porque las especies de este grupo se caracterizan por habitar bosques de dosel cerrado, donde la temperatura es estable y la humedad es alta, lo cual permite la subsistencia de ciertas especies que necesitan este tipo de hábitat. Por este motivo es posible identificar especies características del flanco oriental de la Cordillera Oriental, tales como Allobates cepedai, Allobates sp., Hyalinobatrachium munozorum, Hyloscirtus bogotensis, Rulyrana flavopunctata, Rhaebo glaberrimus, Pristimantis carranguerorum, P. medemi y $O$. carri, y especies cuya distribución incluye los bosques húmedos de la selva amazónica como lo son Dendropsophus minutus, Hypsiboas geographicus, H. lanciformis, Hypsiboas punctatus, Leptodactylus hylaedactylus, Leptodactykus mystaceus, Lithobates palmipes y Rhinella acuminata. La composición taxonómica del piedemonte es, como la definen Lynch, et al., (1997) un ecotono entre los llanos del Orinoco y las selvas de la Amazonía.

Aunque no hay una clara delimitación geográfica de la fauna anfibia llanera, esta se puede definir con base en las especies comunes de áreas abiertas (grupo B) como son Dendropsophus mathiassoni, Elachistocleis ovalis, Physalaemus fischeri, Pseudopaludicola boliviana, P. llanera, Scinax blairi, Scinax kennedyi, S. wandae, Leptodactylus sp. y L. macrosternum), que se caracterizan por habitar las sabanas naturales donde la temperatura es variable y la humedad es baja.

El último grupo (C) está compuesto por especies que habitan bosques de galería (Siphonops annulatus, Osteocephalus taurinus y Leptodactylus lineatus) y por esto su distribución se extiende más allá de los bosques del piedemonte y los bosques húmedos de la selva amazónica, hasta los bosques de galería en las sabanas naturales. 
El conocimiento limitado de la composición y distribución de la herpeto fauna de los municipios que se encuentran en las llanuras constituye un obstáculo para definir la zona de los Llanos Orientales a partir de la composición de las especies de anfibios y reptiles que en ella habitan. Esta dificultad posiblemente se deba a los cambios que han tenido los paisajes naturales de Casanare, que han transformando los bosques nativos del piedemonte en áreas abiertas parecidas a las sabanas, creando, así, las condiciones propicias para el avance de especies "llaneras" hasta el piedemonte. A estos efectos antropogénicos se suma la falta de estudios de herpetofauna en el área de los llanos, cuya situación contrasta con la del piedemonte. Si existe un patrón de distribución de anfibios definido para los llanos, probablemente sea necesario aumentar la escala de los estudios, involucrando el resto de los departamentos que hacen parte de la región de los Llanos Orientales.

Sería de esperar que dada la intensa actividad de explotación de hidrocarburos, sobre todo al sur del departamento (Agencia Nacional de Hidrocarburos, 2013), el desconocimiento sobre estos temas en esta parte del país fuera menor, aunque no son estudios lo que hace falta, sino la divulgación del conocimiento y de los inventarios ya levantados en estas regiones del país.

Como se demostró anteriormente, queda mucho trabajo por hacer para acrecentar el conocimiento de la diversidad real de la herpetofauna de Casanare y aun mucho más para lograr un mejor conocimiento de la distribución de los anfibios. En el caso de los reptiles, el panorama de los estudios sobre su distribución es incluso más oscuro, ya que dicha distribución está muy lejos de conocerse.

\section{Conclusiones}

Debido al relieve de Casanare, con el terreno montañoso de los Andes al occidente y las llanuras al oriente, que constituyen dos unidades eco-geográficas diferentes, la fauna de anfibios y reptiles del departamento es bastante representativa. Por ello, considerando las amplias zonas desconocidas que se identificaron en este estudio, y la predicción obtenida mediante los estimadores de diversidad, se espera que el número de especies en ambos grupos aumente, así como el de los estudios que permitan una mejor aproximación al conocimiento de la diversidad real de Casanare.

Igualmente, se concluyó que la definición de una herpetofauna típicamente llanera es aún difícil de determinar, pues esta generalización debe realizarse a partir de datos que confirmen la existencia de una fauna exclusiva de los Llanos Orientales, lo cual, de momento, no es posible.

A pesar de que el esfuerzo desplegado por el equipo de este estudio se concentró en la zona de los llanos con la intención de lograr una mejor comprensión de su fauna de anfibios y reptiles, quedó claro que debido a su gran extensión y a otras dificultades implícitas en la investigación, es necesario un mayor esfuerzo de muestreo, particularmente para el grupo de los reptiles.

Por último, es necesario llevar a cabo más investigaciones a todo nivel para realimentar los resultados aquí obtenidos, ya que el presente trabajo seguramente está incompleto dada la diversidad esperada para el área de estudio. Sin embargo, este es un punto de partida para generar mayor conocimiento en torno al tema, en beneficio tanto del ecosistema como de los asentamientos humanos presentes en el área de estudio.

\section{Agradecimientos}

Los autores expresan sus agradecimientos a la Universidad Nacional de Colombia, sede Orinoquía, al comité evaluador y organizador de la convocatoria de investigación 2012-II para financiar pasantías y trabajos de grado en pregrado o posgrado en temáticas relacionadas con la Orinoquía, y a la fundación Yoluka por la financiación parcial del proyecto. A los curadores y encargados de las colecciones biológicas de anfibios y reptiles de Colombia, en especial a Andrew Crawford, Museo de Historia Natural, Universidad de los Andes; a Claudia Múnera Roldán, Instituto Alexander von Humboldt; a John D. Lynch y Martha Calderón, Instituto de Ciencias Naturales, Universidad Nacional de Colombia; a José Espitia Barrera, Museo La Salle, Universidad de La Salle y a Julio Mario Hoyos, Museo de Historia Natural, Pontificia Universidad Javeriana, por permitirnos el acceso a los especímenes, y a M. Harvey y R. Caicedo-Portilla por su apoyo en la identificación de especies en grupos de gran complejidad taxonómica. De igual forma, agradecemos muy afectuosamente a los llaneros de los municipios de Aguazul, Paz de Ariporo, Trinidad y San Luis de Palenque, en especial a Ramón Gómez, Juan Carlos Gómez (Chigüi), Esperanza Parales, Abel Antonio Reyes, Andrea, Ana y Cesar Mora, Nilson Gualdrón, Renzo Amaya, Manuel y Marixa Barragán, Esaú Gualdrón, Polo Sandoval, Marina Cruz y Jhon Fredy Fuentes, quienes apoyaron nuestro trabajo en campo. Por último, deseamos agradecer a Pablo Andrés Peña Manrique, Felipe Andrés Aponte y Alejandro Montes por bridarnos su apoyo en la logística durante las salidas de campo.

\section{Conflicto de interés}

Los autores declaran que no tienen ningún conflicto de interés.

\section{Bibliografía}

Acosta-Galvis, A. R. 2000. Ranas, Salamandras y Caecilias (Tetrapoda: Amphibia) de Colombia. Biota Colombiana. 1 (3):289-319.

Acosta-Galvis, A. R., Señaris J. C., Rojas-Runjaic F., RiañoPinzón D. R. 2010. Anfibios y reptiles, pp. 258-289.En Lasso, C. A., J. S. Usma, F. Trujillo, Rial A. Biodiversidad de la cuenca del Orinoco: bases científicas para la 
identificación de áreas prioritarias para la conservación y uso sostenible de la biodiversidad, Instituto de Investigación de Recursos Biológicos Alexander von Humboldt, WWF Colombia, Fundación Omacha, Fundación La Salle e Instituto de Estudios de la Orinoquia (Universidad Nacional de Colombia), Bogotá D. C., Colombia.

Acosta-Galvis, A. R., Alfaro-Bejarano J. P. 2011. Anfibios del Casanare, pp. 139-151. En Usma, J. S., F. Trujillo, Biodiversidad del Casanare: ecosistemas estratégicos del departamento, Gobernación de Casanare-WWF Colombia, Bogotá D.C.

Alfaro, J. P., Acosta-Galvis A. R., Vejarano M. 2011. Reptiles del Casanare, pp. 152-167. En Usma, J. S., Trujillo F.. Biodiversidad del Casanare: ecosistemas estratégicos del departamento, Gobernación de Casanare-WWF Colombia, Bogotá D.C.

Amaral, A. D. 1931. Studies of neotropical ophidia XXIII. Additional notes on Colombian snakes XXVI. Ophidia of Colombia. Bulletin of the Antivenin Institute of America. 4 (4):85-94.

Ayala, S. C. 1986. Saurios de Colombia: lista actualizada y distribución de ejemplares colombianos en los museos. Caldasia. 15: 71-75.

Caicedo-Portilla, R., Dulcey-Cala C. J. 2011. Distribución del gecko introducido Hemidactylus frenatus (Dumeril y Bribon 1836) (Squamata: Gekkonidae) en Colombia. Biota Colombiana. 12 (2 - Especial Simposio Especies Invasoras):45-65.

Campbell, J. A., Lamar W. W. 2004. The venenomous reptiles of the western hemisphere. Comstock Publishing Associates. New York.

Cochran, D. M., Goin C. J. 1970. Frogs of Colombia. Smithsonian institution Press. Washington.

Cortés, J., Sánchez P. 2011. Mamíferos, reptiles y ecosistemas del bloque Cubiro (Casanare): educación ambiental para la conservación. Instituto de Estudios Ambientales, Universidad Nacional de Colombia. Alange Energy Corp. Bogotá.

Faivovich, J., Haddad C. F. B., García P. C. A., Frost D. R., Campbell J. A., Wheeler W. C. 2005. Systematic review of the frog family Hylidae, with special reference to Hylinae : Phylogenetic analysis and taxonomic revision. Bulletin of the American Museum of Natural History. 294 1-240.

Frost, D. R., Grant T., Faivovich J., Bain R. H., Haas A., Haddad C. F. B., De Sa R. O., Channing A., Wilkinson M., Donnellan S. C., Raxworthy C. J., Campbell J. A., Blotto B. L., Moler P., Drewes R. C., Nussbaum R. A., Lynch J. D., Green D. M. \& Wheeler W. C. 2006. The amphibian tree of life. Bulletin of the American Museum of Natural History. 297 1-370.

Grant, T., Frost D. R., Caldwell J. P., Gagliardo R., Haddad C. F. B., Kok P. J. R., Means D. B., Noonan B. P., Schargel W. E., Wheeler W. C. 2006. Phylogenetic systematics of dart- poison frogs and their relatives (Amphibia, Athesphatanura, Dendrobatidae). Bulletin of the American Museum of Natural History. 299 1-262.

Harvey, M. B., Ugueto G. N.. Gutberlet J. 2012. Review of teiid morphology with a revised taxonomy and phylogeny of the Teiidae (Lepidosauria: Squamata). Zootaxa. 3459 1-156.

Hedges, S. B., Duellman W. E., Heinicke M. P. 2008. New World direct-developing frogs (Anura: Terrarana): Molecular phylogeny, classification, biogeography, and conservation. Zootaxa. 1737 1-182.

Heyer, W. R. 1969. The adaptive ecology of the species groups of the genus Leptodactylus (Amphibia, Leptodactylidae). Evolution. 23 (3):421-428.

Lehr, E. 2002. Amphibien und Reptilien in Peru: die Herpetofauna entlang des 10. Breitengrades von Peru: Arterfassung, Taxonomie, ökologische Bemerkungen und biogeographische Beziehungen. Natur und Tierverlag. Münster, Germany.

Ludwing, J. A., Reynolds J. F. 1988. Statistical Ecology (A Primer on Methods and Computing). Wiley Interscience Publication. USA.

Lynch, J. D., Ruiz-Carranza P. M., Ardila-Robayo M. C. 1997. Biogeographic patterns of Colombian frogs and toads. Rev. Acad. Colomb. Cienc. Ex. Fis. Nat. 21 (80):237-248.

Lynch, J. D. 2006. The amphibian fauna in the Villavicencio region of eastern Colombia. Caldasia. 28(1):135-155.

Medem, F. 1965. Bibliografía comentada de reptiles de Colombia. Rev. Acad. Colomb. Cienc. Ex. Fis. Nat. 12(47):299-346.

Medem, F. 1981. Los Cocrodylia de Suramérica. Los Cocrodylia de Colombia. Fondo colombiano de investigaciones científicas y proyectos especiales "Francisco José de Caldas” COLCIENCIAS. Bogotá, Colombia.

Peñuela Recio, L., Castro Lima F., Ocampo-Peñuela N. 2011. Las reservas naturales del nodo Orinoquia en su rol de conservación de la biodiversidad. Fundación Horizonte Verde y Resnatur. Colombia.

Poe, S. 2013. 1986 Redux: New genera of anoles (Squamata: Dactyloidae) are unwarranted. Zootaxa. 3626(2):295-299.

Pyron, R. A., Burbrink F. T., Colli G. R., de Oca A. N. M., Vitt L. J., Kuczynski C. A., Wiens J. J. 2011. The phylogeny of advanced snakes (Colubroidea), with discovery of a new subfamily and comparison of support methods for likelihood trees. Molecular Phylogenetics and Evolution. 58(2):329-342.

Rueda-Almonacid, J. V., Carr L. L., Mittermeier R. A., Rodríguez-Mahecha J. V., Mast R. B., Vogt R. C., Rhodin A. G. J., Ossa-Velásquez J., Rueda J. N., Mittermeier G. 2007. Las tortugas y los cocodrilianos de los países andinos del trópico. Serie de guías tropicales de campo $\mathrm{N}^{\circ}$ 6. Conservación Internacional, Editorial Panamericana, Formas e Impresos. Bogotá, Colombia. 
Rueda, J. V., Castro F., Cortéz C. 2006. Técnicas para el inventario y muestreo de anfibios: una Compilación. 135-172, En Angulo, A., J. V. Rueda-Almonacid, J. V. RodríguezMaecha, E. La Marca. Técnicas de inventario y monitoreo para los anfibios de la región tropical andina, Conservación Internacional. Serie Manuales de Campo $\mathrm{N}^{\circ} 2$, Panamericana Formas e Impresos S.A., Bogotá, Colombia.

Ruiz-Carranza, P. M., Ardila-Robayo M. C., Lynch J. D. 1996. Lista actualizada de la fauna Amphibia de Colombia. Rev. Acad. Colomb. Cienc. Ex. Fis. Nat. 20(77):365-415.
Sánchez-C, Castaño-Mora O. V., Cárdenas, A G. 1987. Diversidad de los reptiles en Colombia. 277-325, En Rangel-Ch, J. O. Colombia Diversidad Biótica I, Instituto de Ciencias Naturales. Editorial Guadalupe, Bogotá, Colombia.

Wüster, W., Yrausquin J. L.. Mijares-Urrutia.2001. A new species of indigo snake from North-Western Venezuela (Serpentes: Colubridae: Drymarchon). Herpetological Journal. 11 157-165.

\section{Anexos}

Anexo 1. Lista de especies de anfibios con registro para el departamento de Casanare, Colombia. Las especies en negrita fueron identificadas como cisandinas.

* Grupo A, †rupo B, *Grupo C

MLS: Museo de La Salle; MUJ: Museo de Historia Natural de la Pontificia Universidad Javeriana; ANDES: Museo de Historia Natural ANDES; IAvH: Instituto Alexander von Humboldt; ICN: Instituto de Ciencias Naturales

\section{AROMOBATIDAE}

Allobates cepedai* NunchÍA, vereda Vega del Tacare, finca La Esperanza, vega del río Tocaria $5^{\circ} 35^{\prime} 8.90^{\prime \prime} \mathrm{N}$, 72'12’29,20” O (IAvH 6371); Aguazul, Inspección de policía de Unete, quebrada Cachiza, finca Buenos Aires $5^{\circ} 11^{\prime} 59,90^{\prime \prime} \mathrm{N}, 72^{\circ} 34^{\prime} 59,95^{\prime \prime}$ O (MLS 1659-64, 1867-8, 1870); Yopal, quebrada el infierno (MLS 1063). Allobates sp.*Aguazul, finca Guarataro (ICN-RPB 233, 236).

\section{BUFONIDAE}

Rhaebo glaberrimus* AguAzul, vereda Cupiagua, quebrada Aguaimara sobre la carretera de Aguazul vía Pajarito $5^{\circ} 10,082^{\prime} \mathrm{N}, 72^{\circ} 38,190^{\prime} \mathrm{O}$ (ICN-TAS 826, 83437, 849, 859-60); NunchíA, vereda Vega del Tacare, finca La Esperanza, vega del rio Tocaria $5^{\circ} 35^{\prime} 8,90^{\prime \prime} \mathrm{N}$, 72'12'29,20" O (IAvH 6710). Rhinella cf. acuminata* Aguazul, vereda El Triunfo (MLS 1820-25, 1834-43, 1871), vereda Cunama, finca La Alborada $5^{\circ} 19^{\prime} 0,12^{\prime \prime} \mathrm{N}$, 72³4'59,95"O (MLS 1891), vereda Cupiagua, quebrada Aguaimara (ICN-TAS 861-62), vereda Manoguia, finca Veracruz $5^{\circ} 10,082^{\prime} \mathrm{N}, 72^{\circ} 38,190^{\prime}$ O (ICN-TAS 814, 863, 874-76); NunchíA (IAvH 7754). Rhinella humboldti Aguazul, vereda El Salitre, finca Brisas del Llano $5^{\circ} 9,37^{\prime}$ N, $72^{\circ} 34,5^{\prime}$ O (MUJ 4515-19, 4689-92), finca Guarataro (ICN-RP 235); Morichal (IAvH 7740-41); NunCHíA, vereda Piedecuesta, finca Las Canarias (IAvH 6711), (IAvH 774246); OrocuÉ, finca La Argentina, bosque de galería del brazo del caño San Miguel (ICN 44607-10), vereda Macucuana, hato El Yopal, Casa del Río Meta $4^{\circ} 57^{\prime} \mathrm{N}, 71^{\circ} 03^{\prime} \mathrm{O}$ (MUJ
5004-16), casco urbano $4^{\circ} 57^{\prime} \mathrm{N}, 71^{\circ} 03^{\prime} \mathrm{O}$ (MUJ 8815), vereda La Esmeralda, finca agroturistica Guarataro (MUJ 8886); PAZ DE ARIPORO, vereda La Colombina, finca El Porvenir, 6²'36,5” N, 71' $34,2^{\circ}$ " O (ICN-TAS 754, 75861); Pore, vereda Matalarga (ICN-RPB 198); SAN LUIS DE PALENQUe, vereda Guaracura, Colegio Emaús 5 ${ }^{\circ} 8^{\prime} 40,4^{\prime \prime} \mathrm{N}$, 70 58'22,6» O (ICN-TAS 459, 527), vereda Guaracura, finca Altamira $5^{\circ} 11^{\prime} 9,77^{\prime \prime} \mathrm{N}, 70^{\circ} 58^{\prime} 53,9^{\prime \prime}$ O (ICN-TAS 532), vereda Guaracura (ICN-TAS 554, 556); TRINIDAD, vereda San Vicente, finca Candalayes $5^{\circ} 18^{\prime} 47,20^{\prime \prime} \mathrm{N}$, $71^{\circ} 22^{\prime} 5,40^{\prime \prime} \mathrm{N}$ (ICN-TAS 253-55, 306), vereda La Cañada, finca La Palmita $5^{\circ} 19^{\prime} 11,4^{\prime \prime} \mathrm{N}, 71^{\circ} 20^{\prime} 51^{\prime \prime} \mathrm{O}$ (ICN-TAS

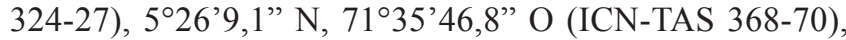
$5^{\circ} 19^{\prime} 11,4^{\prime \prime} \mathrm{N}, 71^{\circ} 20^{\prime} 51^{\prime \prime} \mathrm{O}$ (ICN-TAS 621), 5²6’9,1" N, 71³5'46,8" O (ICN-TAS 640); VIllanueVa, vereda Lecheniel, hacienda Palmas del Casanare $4^{\circ} 57^{\prime} \mathrm{N}, 73^{\circ} 94^{\prime}$ O (MUJ 8241-43, 8649-53), Palmera del Oriente (ICNJDL 30137). Rhinella marina Aguazul, vereda El Salitre, finca Brisas del Llano $5^{\circ} 9,37^{\prime} \mathrm{N}, 72^{\circ} 34,5^{\prime} \mathrm{O}$ (MUJ 4683, 4684-85, 6205); NunchíA, vereda Piedecuesta, finca Las Canarias (IAvH 6712); OROCUÉ, vereda Macucuana, hato El Yopal, Casa del Río Meta $4^{\circ} 57^{\prime} \mathrm{N}, 7^{\circ} 03^{\prime} \mathrm{O}$ (MUJ 540003), (MUJ 8843-49), casco urbano (MUJ 8850-56, 8931), vereda La Esmeralda, finca agroturística Guarataro (MUJ 8857-58); SAn Luis de PAlenque, vereda Guaracura, Colegio Emaús 5 $8^{\circ} 40,4^{\prime \prime} \mathrm{N}, 70^{\circ} 58^{\prime} 22,6^{\prime \prime} \mathrm{O}$ (ICN-TAS 457), vereda Guaracura (ICN-TAS 469), Morichal (IAvH 6658).

\section{CAECILIIDAE}

Siphonops annulatus ${ }^{*}$ VIllanUeVA, vereda El Encanto, finca Río Grande (ICN-JDL 30179-83)

\section{CENTROLENIDAE}

Rulyrana flavopunctata* AgUAZUL, vereda Cupiagua, quebrada Hoyo Caliente $5^{\circ} 14,026^{\prime} \mathrm{N}, 72^{\circ} 40,298^{\prime}$ O (ICNTAS 878-880).

\section{HYLIDAE}

Dendropsophusmathiassoni ${ }^{\dagger}$ AguAZul (IAvH 7637-38), 
finca Grismania (ICN-RPB 221-24), vereda Cupiagua, finca de Antonio Munévar (MLS 1786-91), vereda El Salitre, finca Brisas del Llano 5'9,37' N, 72³4,5' O (MUJ 3787-88, 3790-91, 4561, 4564-65, 4723-32, 6206-07, 6210-24); Morichal (IAvH 7634-36), NunchÍA, vereda Vega del Tacare, finca La Esperanza, vega del rio Tocaria $5^{\circ} 35^{\prime} 8,90^{\prime \prime} \mathrm{N}$, 72¹2’29,20” O (IAvH 6717-21); OrocuÉ (MUJ 8946-49), desviación a los módulos desde la carretera (ICN 44621-25), pozo Tambaqui 1, 17,5 $\mathrm{Km}$ en línea recta desde OrocuÉ (ICN 44616-20), reserva Wisirare, municipio Orucué (ANDES-A 1144); PAZ dE ArIPoro, vereda La Colombina, entre finca El Porvenir y El Baile (ICN-TAS 791-93); PORE, vereda Matalarga $05^{\circ} 27^{\prime} 34$ " N, 071 $41^{\prime}$ '46" O (ICN-RPB 191-93); SAN Luis de PAlenQue (ICN 2639-43), vereda Guaracura 5 ${ }^{\circ} 11^{\prime} 9,77^{\prime \prime} \mathrm{N}, 70^{\circ} 58 ' 53,9$ ' O (ICN-TAS 467, 470-76, 553), vereda Guaracura, Colegio Emaús 58'40,4” N, 7058'22,6” O (ICN-TAS 204), vereda Guaracura, finca Altamira 5'11'9,77' N, 7058'53,9” O (ICN-TAS 536); Trinidad, vereda La Cañada, finca La Palmita $5^{\circ} 19^{\prime} 11,4$ ” N, 71'20'51" O (ICN-TAS 606, 625-28, 652), vereda San Vicente, Caño Osorio $5^{\circ} 18^{\prime} 47,20^{\prime \prime}$ N, 71²2'5,40” O (ICNTAS 257), vereda San Vicente, finca Candalayes $5^{\circ} 18^{\prime} 47,20^{\prime \prime}$ N, 71²2'5,40" O (TAS 598-99); VillanUEVA, vereda Lecheniel, hacienda Palmas del Casanare $4^{\circ} 57^{\prime} \mathrm{N}, 7^{\circ} 94^{\prime} \mathrm{O}$ (MUJ 8659-62), Palmeras de Oriente (ICN-JDL 30127, 30169, 30170); Yopal, vereda El Milagro, entre Morichal y Tilodirán, finca de Julio Salcedo $5^{\circ} 11$ '34” N, 72 18 ' 43 ” O (ICN-JDL 22867). Dendropsophus minutus* AguAZul, Cupiagua 5¹9’0,12” N, 72³4'59,95” O (MLS 1899-04), vereda Cupiagua, finca de Antonio Munévar (MLS 1798, 1808), vereda Cunama. finca La Alborada 5'19’0,12” N, 72॰34'59,95” O (MLS 1878-81), vereda El Salitre, finca Brisas del Llano $5^{\circ} 9,37^{\prime} \mathrm{N}, 72^{\circ} 34,5^{\prime} \mathrm{O}$ (MUJ 4548-51, 4572, 4733-37), vereda El Triunfo 5¹9'0,12” N, 72॰34'59,95” O (MLS 1809-13, 1848-52, 1915-22); NunchÍA (IAvH 7768), vereda Piedecuesta, finca Las Canarias (IAvH 6724-28), vereda Vega del Tacare, finca La Esperanza, vega del río Tocaria 5'35'8,90” N, 72¹2’29,20” O (IAvH 6722-23). Hypsiboas boans AguAzul, vereda Unete, quebrada La Vegana (ICN 36384), vereda Cunama, finca La Alborada 5०19'0,12” N, 72³4'59,95” O (MLS 1888), vereda Cupiagua, quebrada Aguaimara sobre la carretera de Aguazul vía Pajarito (ICN-TAS 829), vereda Manoguía, finca Veracruz (ICN-TAS 867, 869, 870-72); 5¹9'0,12” N, 72॰34'59,95” O (MLS 763). Hypsiboas crepitans AGuAzul, finca Grismania (ICN-RPB 218), finca Guarataro, (ICN-RPB 228), vereda El Salitre, finca Brisas del Llano $5^{\circ} 9,37^{\prime} \mathrm{N}$, 72³4,5’ O (MUJ 3783-86, 4539-40, 4566, 4700-05, 512226, 5159, 6194-01); vereda El Triunfo 5¹9’0,12” N, 72॰34'59,95” O (MLS 1814-19, 1844-47, 1912-14); NunchíA, Hato Venecia (IAvH 4917), vereda Vega del Tacare, finca La Esperanza, vega del río Tocaria $5^{\circ} 35^{\prime} 8,90^{\prime \prime} \mathrm{N}$, 72¹2’29,20” O (IAvH 6714); Orocué (MUJ 8944), casco urbano, centro turístico vacacional Daingo (ICN 44611-15), Reserva Wisirare, municipio de Orucué (ANDES-A-AJC 2321), vereda El Triunfo 5 $5^{\circ} 19^{\prime} 0,12^{\prime \prime} \mathrm{N}, 72^{\circ} 34^{\prime} 59,95 " \mathrm{O}$ (MLS 1321-23), vereda Macucuana, hato El Yopal, Casa del Río Meta (MUJ 5056-58), PAZ DE ARIPORO, vereda La Colombina, finca El Porvenir $6^{\circ} 2^{\prime} 36,5^{\prime}$ N, 71 ${ }^{\circ}$ '34,2” O (ICN-TAS 756, 766-70), vereda La Colombina, entre finca El Porvenir y El Baile (ICN-TAS 787-90); Pore, vereda Matalarga (ICN-RPB 183-194); SABANALARGA (ANDES-A 1063); SAN LuIS de PALENQUe (ICN 2638); vereda Guaracura (ICN-TAS 465-66, 477); vereda Guaracura, Colegio Emaús $5^{\circ} 8^{\prime} 40,4 " \mathrm{~N}, 70^{\circ} 58^{\prime} 22,6^{\prime \prime} \mathrm{O}$ (ICN-TAS 169, 205, 439, 443, $444-47,551$ ), vereda La Lucha $5^{\circ} 23^{\prime} 15,0^{\prime \prime} \mathrm{N}, 71^{\circ} 37^{\prime} 16,99^{\prime \prime} \mathrm{O}$ (ICN-TAS 421-22); TRINIDAD, vereda La Cañada, finca El Mirador 5²6’9,1" N, 71³5'46,8' O (ICN-TAS 371, 642),

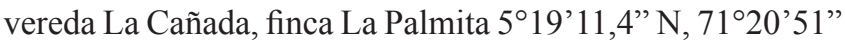
O (ICN-TAS 613, 622, 653), vereda San Vicente, finca Candalayes $5^{\circ} 18^{\prime} 47,20^{\prime \prime} \mathrm{N}, 71^{\circ} 22^{\prime} 5,40^{\prime \prime} \mathrm{O}$ (ICN-TAS 258, 282, 313, 564, 580); VillanUEVA $5^{\circ} 19^{\prime} 0,12^{\prime \prime} \mathrm{N}, 72^{\circ} 34^{\prime} 59,95^{\prime}$ " O (MLS 1456), Palmera del Oriente (ICN-JDL 30125, 30128-30, 30138, 30155-56, 30174), vereda Lecheniel,

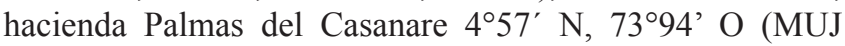
8238-40); YoPAL, vereda El Milagro, entre Morichal y Tilodirán, finca de Julio Salcedo $5^{\circ} 11^{\prime} 34^{\prime \prime}$ N, 72 $18^{\prime}-43^{\prime \prime} \mathrm{O}$ (ICN-JDL 22866); Morichal (IAvH 6659-61). Hypsiboas geographicus* AgUAZUL, vereda Manoguía, finca Veracruz $5^{\circ} 10,082^{\prime}$ N, 72³8,190 O (ICN-TAS 821, 865). Hypsiboas lanciformis* AguAZuL, vereda Cupiagua, La Palmicha 5¹9'0,12” N, 72॰34'59,95" O (MLS 1658, 1905), vereda Cupiagua, quebrada Aguaimara (ICN-TAS 848, 851-52), vereda El Salitre, finca Brisas del Llano $5^{\circ} 9,37^{\prime} \mathrm{N}, 72^{\circ} 34,5^{\prime}$ O (4536-38); NunchíA, vereda Vega del Tacare, finca La Esperanza, vega del río Tocaria $5^{\circ} 35^{\prime} 8,90^{\prime \prime} \mathrm{N}, 72^{\circ} 12^{\prime} 29,20^{\prime}$ ” O (IAvH 6715-16); SABANALARGA (ANDES-A-AJC 3971, 3975). Hypsiboas punctatus* (ICN-TAS 892); AGUAZUL, Cupiagua $5^{\circ} 19^{\prime} 0,12^{\prime \prime} \mathrm{N}, 72^{\circ} 34^{\prime} 59,95^{\prime}$ ' O (MLS 1896-98), finca Grismania (ICN-RPB 214, 216-20), quebrada Costa Grande a $100 \mathrm{~m}$ del retén (ICN 9548-51), vereda Cupiagua, finca de de Antonio Munévar 5¹9’0,12” N, 72³4'59,95” O (MLS 1797), vereda Cunama, finca La Alborada 5¹9'0,12”, N, 72³4'59,95" O (MLS 1873-76), vereda Cupiagua, quebrada Aguaimara (ICN-TAS 850, 853-854), vereda Cupiagua, quebrada Aguaimara sobre la carretera de AGUAZUL vía Pajarito $5^{\circ} 10,082^{\prime} \mathrm{N}, 72^{\circ} 38,190^{\prime} \mathrm{O}$ (ICN-TAS 823), vereda El Salitre, finca Brisas del Llano $5^{\circ} 9,37^{\prime} \mathrm{N}, 72^{\circ} 34,5^{\prime}$ O (MUJ 3792-93, 4456-57, 4706-12, 4752, 5145, 48, 622529), vereda El Triunfo 5०19'0,12” N, 72॰34'59,95” O (MLS 1853-54, 1911), vereda Manoguía, finca Veracruz $5^{\circ} 10,082$ ' N, 72³8,190’ O (ICN-TAS 877); SABANALARGA (ANDES-A 1042-44, 1049, ANDES-A-AJC 4100); VillanUEVA, vereda Lecheniel, hacienda Palmas del Casanare $4^{\circ} 57^{\prime} \mathrm{N}, 73^{\circ} 94^{\prime} \mathrm{O}$ (MUJ 8246) Palmas de Oriente (ICN-JDL 30173). Hyloscirtus bogotensis* LA SALINA, El Arenal, quebrada ca. 
Km 108 Socha, La Punta (ICN 5811, 5853). Osteocephalus carri* AguAZuL, vereda Cupiagua, quebrada Hoyo Caliente 5¹4,026’ N, 7240,298’ O (ICN-TAS 881-82, 890).

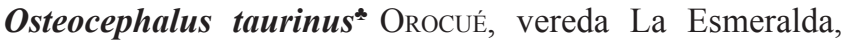
finca agroturística Guarataro (MUJ 8814). Phyllomedusa hypochondrialis ${ }^{\dagger}$ AguAZuL, vereda El Salitre, finca Brisas del Llano $5^{\circ} 9,37^{\prime} \mathrm{N}, 72^{\circ} 34,5^{\prime} \mathrm{O}$ (MUJ 4520-33, 4693-77, 6203-04), vereda El Triunfo (MLS 1805); Morichal (IAvH 7735-38); NunchíA (IAvH 7732-34), vereda Vega del Tacare, finca La Esperanza, vega del río Tocaria (IAVH 7017); Orocué, casco urbano, centro turístico vacacional Daingo (ICN 44647-48); Pore, vereda Matalarga (ICN-RPB 20210); San Luis de Palenque, vereda Guaracura, Colegio Emaús 58'40,4" N, 7058'22,6" O (ICN-TAS 430-31, 55962); TRINIDAD, vereda La Cañada, finca La Palmita 5¹9'11,4”, N, 71²0'51" N (ICN-TAS 612, 619); Villanueva, Palmera Santana (ICN-JDL 30115-16), Palmera del Oriente (ICNJDL 30331); YoPAL, vereda El Milagro, entre Morichal y Tilodirán, finca de Julio Salcedo $5^{\circ} 11^{\prime} 34^{\prime \prime}$ N, 72¹8'43” O (ICN-JDL 22668-69). Pseudisparadoxa AguAzul, finca Grismania (ICN-RPB 227), Morichal (IAvH 7767, 777478); SAN Luis de PAlENQUe, vereda Guaracura (ICN-TAS 478), vereda Guaracura, finca Altamira 5'11'9,77' $\mathrm{N}$, 7058'53,9” O (ICN-TAS 513, 516-23); TRINIDAD, vereda La Cañada, finca La Palmita $5^{\circ} 19^{\prime} 11,4^{\prime \prime} \mathrm{N}, 71^{\circ} 20^{\prime} 51^{\prime \prime} \mathrm{O}$ (ICNTAS 611, 617, 629, 641); YopAL, vereda El Milagro, entre Morichal y Tilodirán, finca de Julio Salcedo (ICN-JDL 22870-71). Scinax blairi ${ }^{\dagger}$ OROCUE, Reserva Wisirare (ANDES-A-AJC 2312); SABANALARGA (ANDES-A 1067 , 1071-72, ANDES-A-AJC 4120), en charco artificial, Sabanalarga (AJC 1068). Trinidad, vereda La Cañada, finca La Palmita 5¹9'11,4” N, 71²0'51" O (ICN-TAS 162, 616). Scinax kennedyi (ANDES-A 1060), OROCUÉ, pozo Tambaquí y morichal aledaño (ICN 44626-35); SABANALARGA (ANDES-A 1051, 1058, 1061-62, 1066, 1084, ANDES-AAJC 4058). Scinax rostratus Aguazul, vereda Cupiagua, finca de Antonio Munévar (MLS 1792-96), vereda El Salitre, finca Brisas del Llano 59,37’ N, 72³4,5' O (MUJ 4541, 4751); Morichal (IAvH 7726-27); NunchíA (IAvH 7725), vereda Piedecuesta, finca Las Canarias (IAvH 7009-11); Orocué (MLS 1322), vereda Macucuana, hato El Yopal,

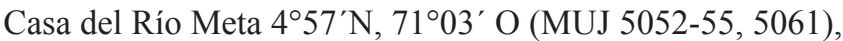
casco urbano (MUJ 8817); Pore, vereda Matalarga (ICNRPB 184-90; SAN LUIS DE PALENQUE, vereda Bocas del Ulere, finca Altamira 5 ${ }^{\circ} 11^{\prime} 9,77^{\prime}$ N N, 7058'53,9”' O (ICN-TAS 237, 240), vereda Guaracura, Colegio Emaús 58'40,4" $\mathrm{N}$, 7058'22,6" O (ICN-TAS 224), SAN Luis de PAlenque, vereda Guaracura, finca Altamira 5 $5^{\circ} 11^{\prime} 9,77^{\prime \prime}$ N, 7058'53,9" O (ICN-TAS 511-12, 534-35); TRINIDAD, vereda San Vicente, finca Candalayes 5 $5^{\circ} 18^{\prime} 47,20^{\prime \prime} \mathrm{N}, 71^{\circ} 22^{\prime} 5,40^{\prime}$ O O (ICN-TAS 341), vereda La Cañada, finca El Mirador 5²6’9,1" $\mathrm{N}$, 71 35 '46,8” O (ICN-TAS 427), vereda La Cañada, finca. La Palmita $5^{\circ} 19^{\prime} 11,4^{\prime \prime} \mathrm{N}, 7^{\circ} 20^{\prime} 51^{\prime \prime} \mathrm{O}$ (ICN-TAS 625);
VillanueVA, casco urbano (ICN-JDL 30132). Scinaxruber Aguazul, vereda Cunama (MLS 1882), vereda El Salitre, finca Brisas del Llano 59,37’ N, 72³4,5’ O (MUJ 454445), finca Grismania (ICN-RPB 213, 215), Morichal (IAvH 7728-30); NunchíA (IAvH 7731), hato Venecia (IAvH 489810); Orocué, casco urbano (MUJ 8884), vereda Macucuana, hato El Yopal, Casa del Río Meta (MUJ 5051), Reserva Wisirare, Municipio de Orucué (ANDES-A-AJC 2314-15, 2317, 2324-25); SABANALARGa (ANDES-A 1040, 1046, 1064-65); SAN Luis de Palendue, vereda Guaracura, colegio EMAUS 5 $8^{\prime} 40,4^{\prime \prime}$ N, 7058'22,6" O (ICN-TAS 165, 557 58 ), vereda La Riverita, finca La Bretaña $5^{\circ} 8^{\prime} 41,2^{\prime \prime} \mathrm{N}$, 7057'27,9" O (ICN-TAS 231), vereda Bocas del Ulere, finca Altamira $5^{\circ} 11^{\prime} 9,77^{\prime \prime} \mathrm{N}, 70^{\circ} 58$ '53,9” O (ICN-TAS 236, 238-39), vereda La Lucha 5²3'15,0” N, 71³7’16,9” O (ICN-TAS 423); TRINIDAD, vereda San Vicente, finca Candalayes $5^{\circ} 18^{\prime} 47,20^{\prime}$ N, 71²2'5,40” O (ICN-TAS 312), vereda La Cañada, finca San Miguel 5'24'49,8” $\mathrm{N}$, 71'36'38,4" O (ICN-TAS 394-95); VillanueVA, vereda Lecheniel, hacienda Palmas del Casanare $4^{\circ} 57^{\prime} \mathrm{N}, 73^{\circ} 94^{\prime} \mathrm{O}$ (MUJ 8237, 8654-58); Yopal, vereda El Milagro, entre Morichal y Tilodirán, finca de Julio Salcedo $5^{\circ} 11>34 » \mathrm{~N}$, $72^{\circ} 18>43 »$ O (ICN-JDL 22863). Scinax sp. ${ }^{\dagger}$ SAN LUIS DE PALENQUE, vereda Guaracura, finca Altamira 5'11'9,77' $\mathrm{N}$, 7058'53,9" O (ICN-TAS 514). Scinax wandae ${ }^{\dagger}$ AGUAZUL, vereda El Triunfo (MLS 1923), vereda El Salitre, finca Brisas del Llano 59,37’ N, 72³4,5’ O (MUJ 3794, 4552, 6202); Nunchía (IAvH 7762-66), vereda Vega del Tacare, finca La Esperanza, vega del río Tocaria $5^{\circ} 35^{\prime} 8,90^{\prime \prime} \mathrm{N}, 72^{\circ} 12^{\prime} 29,20^{\prime \prime}$ O (IAvH 7018); Orocué (MLS 775, MUJ 8945), borde de la carretera Orocué-Yopal, aproximadamentea $8 \mathrm{~km}$ desde Orocué (ICN 44636-42), casco urbano de Orocué $4^{\circ} 57^{\prime} \mathrm{N}$, 7103' O (MUJ 8885), desviación a los módulos desde la carretera Orocué-Yopal (ICN 44643-46), vereda Macucuana, hato El Yopal, Casa del Río Meta 457’ N, 7103' O (MUJ 5062-80); PAZ DE ARIPORO, vereda La Colombina, finca El Porvenir 6²’36,5” N, 71'5’34,2” O (ICN-TAS 743-47), vereda La Colombina, entre finca El Porvenir y El Baile (ICN-TAS 794-95); SAN LuIS DE PALENQUe, vereda Bocas del Ulere, finca Altamira 5०11'9,77' N, 7058'53,9” O (ICNTAS 241), vereda Guaracura, Colegio Emaús 58'40,4" $\mathrm{N}$, 7058'22,6" O (ICN-TAS 461); TAURAMENA (MLS 767); TRINIDAD, vereda La Cañada, finca La Palmita $5^{\circ} 19^{\prime} 11,4^{\prime \prime} \mathrm{N}$, 71²0'51" O (ICN-TAS 654-55); VilLANUEVA, casco urbano (ICN-JDL 30133, 30134, 30158), Palmas del Oriente (ICNTAS 30172), vereda El Encanto, finca Río Grande (ICN-JDL 30164), vereda Lecheniel, hacienda Plantas del Casanare 457'N, 7394' o (MUJ 8247); Yopal, vereda El Milagro, entre Morichal y Tilodirán, finca de Julio Salcedo $5^{\circ} 11^{\prime} 34^{\prime \prime}$ N; 72॰18'43" O (ICN-JDL 22864-65). Scinax $\boldsymbol{x}$-signata NunCHÍA, vereda El Milagro, entre Morichal y Tilodirán, finca de Julio Salcedo 5¹1'34" N, 72¹8'43" O (IAvH 7019); SAN LuIS DE PALENQue (ICN 2644-59). Trachycephalus 
typhonius (IAvH 6657), Morichal (IAvH 6657); OrocuÉ, vereda Macucuana, hato El Yopal, Casa del Río Meta 457' N, 71 ${ }^{\circ} 03^{\prime}$ O (MUJ 5043-50); PAZ De Ariporo, vereda La

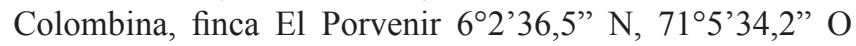
(ICN-TAS 752-53, 771-81), vereda La Colombina, entre finca El Porvenir y El Baile (ICN-TAS 809); SAN LuIS DE PALENQUe, vereda Guaracura, finca Altamira $5^{\circ} 11^{\prime} 9,77^{\prime \prime} \mathrm{N}$, $70^{\circ} 58^{\prime} 53,99^{\prime \prime} \mathrm{O}$ (ICN-TAS 515, 540, 543, 547); TAURAMENA (MLS 766).

\section{LEPTODACTYLIDAE}

Engystomops pustulosus (ICN.TAS 883); PAZ DE ARIPORo, vereda La Colombina, finca El Porvenir $6^{\circ} 2 ’ 36,5^{\prime \prime} \mathrm{N}$, 71'5'34,2” O (ICN-TAS 736-41, 762-65). Leptodacty-lus colombiensis (ICN-TAS 884); Aguazul, Cupiagua $5^{\circ} 19^{\prime} 0,12^{\prime \prime} \mathrm{N}, 72^{\circ} 34^{\prime} 59,95^{\prime \prime} \mathrm{O}$ (MLS 1906), hacienda La Esperanza (ICN 9561-63), vereda Cunama, finca La Alborada 5०19'0,12” N, 72³4'59,95” O (MLS 1890), vereda Cupiagua, vereda Aguaimara sobre la carretera de Aguazul vía Pajarito $5^{\circ} 10,082^{\prime}$ N, $72^{\circ} 38,190^{\prime}$ O (ICN-TAS 824-25, 830-33), vereda El Triunfo $5^{\circ} 19^{\prime} 0,12^{\prime \prime} \mathrm{N}$, 72³4'59,95” O (MLS 1668-70, 1803-04, 1807, 1826, 1927-28, 1930), vereda Manoguía, finca Veracruz 5¹0,082' $\mathrm{N}, 7^{\circ} 38,190^{\prime}$ O (ICN-TAS 873); NunchíA, vereda Piedecuesta, finca Las Canarias (IAvH 7012); OrocuÉ (MUJ 8941), finca La Argentina, bosque de galería del brazo del caño San Miguel (ICN 44655-60), vereda La Esmeralda, finca agroturística Guarataro (MUJ 8860-83); Paz de Ariporo, vereda La Colombina, finca El Porvenir ${ }^{\circ} 2^{\prime} 36,5^{\prime}$ N N, 715'34,2” O (ICN-TAS 725), vereda La Colombina, entre finca El Porvenir y El Baile (ICN-TAS 810-12), Sabanalarga (ANDES-A 1083); San Luis de PALENQUe, vereda Guaracura, colegio EMAUS 5 $8^{\prime} 40,4$ " N, 70 58'22,6” O (ICN-TAS 207), vereda Guaracura, finca Altamira $5^{\circ} 11^{\prime} 9,77^{\prime}$ N $, 70^{\circ} 58^{\prime} 53,9^{\prime \prime} \mathrm{O}$ (ICN-TAS 529-30, $538,541-42,545)$, vereda La Lucha $5^{\circ} 23^{\prime} 15,0^{\prime \prime} \mathrm{N}$, 71'37'16,9" O (ICN-TAS 419); TRINIDAD, vereda La Cañada, finca El Mirador $5^{\circ} 26^{\prime} 9,1^{\prime \prime} \mathrm{N}, 71^{\circ} 35^{\prime} 46,8^{\prime \prime} \mathrm{O}$ (ICN-TAS 367), vereda La Cañada, finca San Miguel (ICNTAS 409), vereda San Vicente, finca Candalayes $5^{\circ} 18^{\prime} 47,20^{\prime \prime}$ N, 71²2'5,40 O” (ICN-TAS 267); Villanueva, vereda Lecheniel, hacienda Palmas del Casanare. N 4 ${ }^{\circ} 57^{\prime}$, O 73⒐' (MUJ 8248-49). Leptodactylus fragilis (ICN-TAS 883); Orocué, (MUJ 8937-38), casco urbano, centro turístico vacacional Daingo (ICN 44649); PAZ DE ARIPORO, vereda La Colombina, finca El Porvenir N 6²'36.5”, O 71''34.2" (ICN-TAS 735); PORE, vereda Matalarga (ICNRPB 197, 199); SAN Luis De Palenque, vereda Guaracura (ICN-TAS 468, 487-91), vereda Guaracura, Colegio EMAUS 5 $8^{\prime} 40,4^{\prime \prime} \mathrm{N}, 70^{\circ} 58^{\prime} 22,6^{\prime \prime} \mathrm{O}$ (ICN-TAS 438, 44042, 458, 460, 462-63, 497-09), vereda Guaracura, finca Altamira $5^{\circ} 11^{\prime} 9,77^{\prime}$ N $, 70^{\circ} 58^{\prime} 53,9^{\prime \prime}$ O (ICN-TAS 510, $522,544)$, vereda La Lucha $5^{\circ} 23^{\prime} 15,0^{\prime \prime} \mathrm{N}, 71^{\circ} 37^{\prime} 16,9^{\prime \prime} \mathrm{O}$
(ICN-TAS 417-18); TRINIDAD, vereda La Cañada, finca El Mirador $5^{\circ} 26^{\prime} 9,1^{\prime \prime} \mathrm{N}, 71^{\circ} 35^{\prime} 46,8^{\prime \prime} \mathrm{O}$ (ICN-TAS 356-62, 366, 384, 634, 637); TRINIDAD, vereda La Cañada, finca La Palmita $5^{\circ} 19^{\prime} 11,4^{\prime \prime} \mathrm{N}, 71^{\circ} 20^{\prime} 51^{\prime \prime} \mathrm{O}$ (ICN-TAS 603, 644), vereda La Cañada, finca San Miguel $5^{\circ} 24^{\prime} 49,8^{\prime \prime} \mathrm{N}$, 71 ${ }^{\circ} 36^{\prime} 38,4^{\prime \prime}$ O (ICN-TAS 398-00, 402-07), vereda San Vicente, caño Osorio $5^{\circ} 18^{\prime} 47,20^{\prime \prime} \mathrm{N}, 71^{\circ} 22^{\prime} 5,40^{\prime \prime}$ O (ICNTAS 328-330), vereda San Vicente, finca Candalayes $5^{\circ} 18^{\prime} 47,20^{\prime \prime} \mathrm{N}, 71^{\circ} 22^{\prime} 5,40^{\prime \prime} \mathrm{O}$ (ICN-TAS 259-64), 279-80, 307-09, 318-21, 323, 567, 681, 586-97), Villanueva, Palmera Santana (ICN-JDL 30117), vereda El Encanto, finca Río Grande (ICN-JDL 30185-87). Leptodactylis fuscus Aguazul, finca Grismania (ICN-RPB 225), vereda Cunama, finca La Alborada 5'19'0,12” N, 72³4'59,95” O (MLS 1883), vereda Manoguia, finca Veracruz $5^{\circ} 10,082^{\prime}$ N, $72^{\circ} 38,190^{\prime}$ O (ICN-TAS 815-16), vereda Salitre, finca Brisas del Llano $5^{\circ} 09,37^{\prime} \mathrm{N}, 72^{\circ} 34,58$ O (MUJ 4542-43, 4713-20); Morichal (IAvH 7680); Nunchía (IAvH 768184), vereda Vega del Tacare, finca La Esperanza, vega del río Tocaria $5^{\circ} 35^{\prime} 8,90^{\prime \prime} \mathrm{N}, 72^{\circ} 12^{\prime} 29,20^{\prime \prime} \mathrm{O}$ ( IAvH 7020, 7681-84); Orocué (ICN 44650-54), casco urbano de Orocué $4^{\circ} 57^{\prime} \mathrm{N}, 7^{\circ} 03^{\prime} \mathrm{O}$ (MUJ 8801-03), vereda La Esmeralda, finca agroturística Guarataro (MUJ 8804-13), OROCUÉ, vereda Macucuana, hato El Yopal, Casa del Río Meta 457'

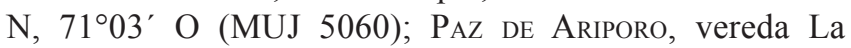
Colombina, entre finca El Porvenir y El Baile (ICN-TAS 799-08), vereda La Colombina, finca El Porvenir 6²'36,5” $\mathrm{N}, 7^{\circ} 5^{\prime} 34,2^{\prime \prime} \mathrm{O}$ (ICN-TAS 726-33); Pore, vereda Matalarga (ICN-RPB 195-96); SABANALARGA (ANDES-A 1052, 1057, 1059, ANDES-A-AJC 4088); SAN LUIS DE PALENQUE, vereda Guaracura (ICN-TAS 464, 479-83), vereda Guaracura, Colegio Emaús 5 8 8' 40,4” N, 70 58'22,6" O (ICN-TAS 208-09, 227, 496), vereda Guaracura, finca Altamira $5^{\circ} 11^{\prime} 9,77^{\prime}$ N, $70^{\circ} 58$ '53,9” O (ICN-TAS 531); Trinidad, N vereda La Cañada, finca El Mirador $5^{\circ} 26^{\prime} 9,1^{\prime \prime}$ $\mathrm{N}, 71^{\circ} 35^{\prime} 46,8^{\prime \prime} \mathrm{O}$ (ICN-TAS 349, 363, 385, 635), vereda La Cañada, finca La Palmita $5^{\circ} 19^{\prime} 11,4^{\prime \prime} \mathrm{N}, 71^{\circ} 20^{\prime} 51^{\prime \prime} \mathrm{O}$ (ICN-TAS 607, 624), vereda La Cañada, finca San Miguel $5^{\circ} 24^{\prime} 49,8^{\prime \prime} \mathrm{N}, 7^{\circ} 36^{\prime} 38,4^{\prime \prime} \mathrm{O}$ (ICN-TAS 396-97, 408), vereda San Vicente, finca Candalayes $5^{\circ} 18^{\prime} 47,20^{\prime \prime} \mathrm{N}$, $71^{\circ} 22^{\prime} 5,40$ ” O (ICN-TAS 246-47, 265-66, 277-78, 304-05, 310, 570, 676-78, 601); Villanueva, Palmas de Oriente (ICN-JDL 30143), vereda El Encanto, finca Río Grande (ICN-JDL 30184), vereda Lecheniel, hacienda Plantas del Casanare $4^{\circ} 57^{\prime} \mathrm{N}, 7^{\circ} 94^{\prime} \mathrm{O}$ (MUJ 8235-36, 8666); Yopal, vereda El Milagro, entre Morichal y Tilodirán, finca de Julio Salcedo $5^{\circ} 11^{\prime} 34^{\prime \prime}$ N, $72^{\circ} 18^{\prime} 43^{\prime \prime}$ O (ICN-JDL 2287475). Leptodactylus hylaedactylus* SABANALARGA (ANDESA 1111); Villanueva, Palmas de Oriente (ICN-JDL 3013941, 30157), Palmera Santana (ICN-JDL 30112-30118-22), vereda Lecheniel, hacienda Palmas del Casanare $4^{\circ} 57^{\prime} \mathrm{N}$, 73⒐' O (MUJ 8253-56). Leptodactylus insularum Morichal (IAvH 8840, 8842, 8844-45); Nunchía (IAvH 
8848); Orocué (MUJ 8939-40), casco urbano de Orocué (MUJ 8798-00), puente sobre el río San Miguel (ICN34683); Sabanalarga (ANDES-A 1109); SAN LuIS DE PAlenque, vereda Guaracura (ICN-TAS 555), vereda Guaracura, caño Osorio 58'40,4” N, 7058'22,6” O (ICNTAS 223), vereda Guaracura, finca Altamira 5 ${ }^{\circ} 11^{\prime} 9,77^{\prime \prime} \mathrm{N}$, 7058' 53,9' O (ICN-TAS 537, 539, 546); TRINIDAD, vereda San Vicente, finca Candalayes $5^{\circ} 18^{\prime} 47,20^{\prime \prime} \mathrm{N}, 71^{\circ} 22^{\prime} 5,40^{\prime \prime}$ O (ICN-TAS 268-69); Leptodactylus lineatus Morichal (IAVH 6655-56); AguAzul, finca Grismania (ICN-RPB 226), vereda La playa, finca de Antonio Munévar (MLS 1672-73), vereda Manoguía, finca Veracruz $5^{\circ} 10,082^{\prime} \mathrm{N}$, 72³8,190’ O (ICN-TAS 864); TRINIDAD, vereda La Cañada, finca La Palmita 5 ${ }^{\circ} 19^{\prime} 11,4$ " N, 71²0'51" O (ICN-TAS 656), vereda La Cañada, finca San Miguel $5^{\circ} 24^{\prime} 49,8^{\prime \prime} \mathrm{N}$, 71'36'38,4” O (ICN-TAS 393), vereda San Vicente, finca Candalayes $5^{\circ} 18^{\prime} 47,20^{\prime \prime} \mathrm{N}, 71^{\circ} 22^{\prime} 5,40$ ” O (ICN-TAS 661); Villanueva, Palmera Santana (ICN-JDL 30113-14), Palmera de Oriente (ICN-JDL 30126), vereda Lecheniel, hacienda Palmas del Casanare $4^{\circ} 57^{\prime} \mathrm{N}, 7^{\circ} 94^{\prime} \mathrm{O}$ (MUJ 8244-45). Leptodactylus macrosternum ${ }^{\dagger}$ HATO COROZAL (MLS 102); Morichal (IAvH 8841, 8843, 8846-47); PAZ DE Ariporo, vereda La Colombina, finca El Porvenir 6²'36,5' N, 715'34,2" O (ICN-TAS 766); SAN LuIS DE PALENQue, vereda Guaracura (ICN-TAS 484-86), vereda Guaracura, Colegio Emaús 5 ${ }^{\circ} 8^{\prime} 40,4 " \mathrm{~N}, 70^{\circ} 58^{\prime} 22,6$ " O (ICN-TAS 206, 225-26, 228), vereda La Lucha $5^{\circ} 23^{\prime} 15,0^{\prime \prime} \mathrm{N}$, 71'37'16,9” O (ICN-TAS 420), vereda La Riverita, finca La Bretaña 5 $8^{\prime} 41,2$ N, 7057'27,9”'O (ICN-TAS 221); TRINIDAD, vereda La Cañada, finca El Mirador 5'26'9, $1^{\prime \prime} \mathrm{N}$, 71'35'46,8” O (ICN-TAS 350-55, 364-65, 383, 386-89), vereda La Cañada, finca La Palmita $5^{\circ} 19^{\prime} 11,4^{\prime \prime} \mathrm{N}$, 71 ${ }^{\circ} 20^{\prime} 51^{\prime \prime}$ O (ICN-TAS 608-10, 614, 618, 620), vereda San Vicente, finca Candalayes $5^{\circ} 18^{\prime} 47,20^{\prime \prime} \mathrm{N}, 71^{\circ} 22^{\prime} 5,40^{\prime \prime} \mathrm{O}$ (ICN-TAS 248-49, 270-76, 281, 311, 563, 568-69, 572-74, 579, 582-85); Yopal, vereda El Milagro, entre Morichal y Tilodirán, finca de Julio Salcedo $5^{\circ} 11>34$ » N , 72॰18’43” O (ICN-JDL 22872-73). Leptodactylus mystaceus* NuNCHÍA, vereda Vega del Tacare, finca La Esperanza, vega del río Tocaria 5³5'8,90" N, 72॰12’29,20” O (IAvH 7021, 7023).

Leptodactylus sp. ${ }^{\dagger}$ TRINIDAD, vereda San Vicente, finca Candalayes $5^{\circ} 18^{\prime} 47,20^{\prime}$ N, 71'22'5,40” O (ICN-TAS 575). Leptodactylus sp. $1^{\dagger} \mathrm{PAZ}$ DE ARIPORO, vereda La Colombina, finca El Porvenir 6 ${ }^{\circ}{ }^{\prime} 36,5^{\prime \prime} \mathrm{N}, 71^{\circ} 5^{\prime} 34,2^{\prime \prime} \mathrm{O}$ (ICN-TAS 734); Physalaemus fischeri ${ }^{\dagger}$ AGUAZUL vereda El Salitre (MUJ 4738-40); Hato Corozal, "La Prevención" (IAvH 1330-3) NunchíA (IAvH 7811-12), vereda Piedecuesta, finca Las Canarias (IAvH 7013). OrocuÉ, (MUJ 8942-3); Reserva Wisirare, municipio de Orocué (ANDES-A-AJC 2309,16); PAZ DE ARIPORO, vereda La Colombina, entre finca El Porvenir y El Baile (TAS 796-8); vereda La Colombina, finca El Porvenir, 6 ${ }^{\circ} 36,5^{\prime}$ ' N, 71'5'34,2” O (TAS 742); PORE, vereda Matalarga; SAN LuIS
De Palenque, vereda Guaracura, Colegio Emaús 58'40,4" N, 7058'22,6" O (ICN-TAS 448); vereda La Lucha 5'23'15,0” N, 71³7'16,9” O (ICN-TAS 416); TAURAMENA (MLS 740) TRINIDAD, vereda La Cañada, finca El Mirador 5²6'9, 1" N, 71³5'46,8" O (ICN-TAS 372-4; TAS 426, 28-29, 636) Pseudopaludicola boliviana ${ }^{\dagger}$ SABANALARGA (ANDES-A 1114-15, 1117, ANDES-A-4115); Yopal, vereda El Milagro, entre Morichal y Tilodirán, finca de Julio Salcedo 5¹1'34” N, 72¹8'43” O (ICN-JDL 22876). Pseudopaludicola llanera ${ }^{\dagger}$ NunchÍA, vereda Piedecuesta, finca Las Canarias (IAvH 7014-15); Orocué (MUJ 895051); PAZ DE ARIPORO, vereda La Colombina, finca El Porvenir 6 ${ }^{\circ} 2^{\prime} 36,5^{\prime}$ "N, 715’34,2” O (ICN-TAS 701-24); SAn Luis de Palenque, vereda Guaracura, colegio Emaús 5 $8^{\prime} 40,4 " \mathrm{~N}, 70^{\circ} 58^{\prime} 22,6 " \mathrm{O}$ (ICN-TAS 208-09, 211-20, 222, 229, 232-33); TRINIDAD, vereda La Cañada, finca El Mirador $5^{\circ} 26^{\prime} 9,1^{\prime \prime} \mathrm{N}, 71^{\circ} 35^{\prime} 46,8^{\prime \prime} \mathrm{O}$ (ICN-TAS 375-82, $632,639)$, vereda San Vicente, caño Osorio $5^{\circ} 18^{\prime} 47,20^{\prime \prime} \mathrm{N}$, 71'22'5,40" O (ICN-TAS 287-03), vereda San Vicente, finca Candalayes $5^{\circ} 18^{\prime} 47,20^{\prime \prime} \mathrm{N}, 71^{\circ} 22^{\prime} 5,40^{\prime}$ O (ICN-TAS 250-52, 342-44, 565-66); VillanUEVA, vereda Lecheniel, hacienda Palmas del Casanare $4^{\circ} 57^{\prime} \mathrm{N}, 73^{\circ} 94^{\prime} \mathrm{O}$ (MUJ 8258).

\section{MICROHYLIDAE}

Elachistocleis ovalis ${ }^{\dagger}$ AGUAZUL, vereda El Salitre, finca Brisas del Llano $5^{\circ} 9,37^{\prime} \mathrm{N}, 72^{\circ} 34,5^{\prime} \mathrm{O}$ (MUJ 4721-22), Morichal (IAvH 7810); Nunchía (IAvH 7811-12), vereda Piedecuesta, finca Las Canarias (IAvH 7016), vereda Sirivania (ICN-JDL 30203); OrocuÉ, casco urbano, centro turístico vacacional Daingo (ICN 44663); PAZ DE ARIPORO, vereda La Colombina, entre finca El Porvenir y El Baile (ICN-TAS 786); SAN LuIS de Palendue (IAvH 7813); Trinidad, vereda La Cañada,

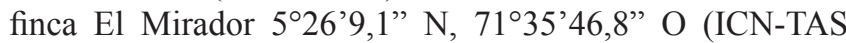
412), vereda La Cañada, finca La Palmita $5^{\circ} 19^{\prime} 11,4^{\prime \prime} \mathrm{N}$, 71'20'51" O (ICN-TAS 623, 658-60), vereda San Vicente, caño Osorio $5^{\circ} 18^{\prime} 47,20^{\prime \prime} \mathrm{N}, 71^{\circ} 22^{\prime} 5,40^{\prime \prime} \mathrm{O}$ (ICN-TAS $331)$, vereda San Vicente, finca Candalayes $5^{\circ} 18^{\prime} 47,20^{\prime \prime} \mathrm{N}$, 71²2'5,40" O (ICN-TAS 256); Villanueva, Palmeras de Oriente (ICN-JDL 31023-24, 30142), vereda El Encanto, finca Río Grande (ICN-JDL 30188-89); YoPAL, vereda El Milagro, entre Morichal y Tilodirán, finca de Julio Salcedo 5'11'34” N, 72¹8'43” O (ICN-JDL 22877).

\section{RANIDAE}

Lithobatespalmipes* (ICN-TAS 893); AguAZul, $1 \mathrm{Km}$ río Cusiana, carretera Pajarito-Agua Azul (ICN 9573-74), Cupiagua (MLS 1907-10), finca Guarataro (ICN-RPB 22932), quebrada Costa Grande a $100 \mathrm{~m}$ del retén (ICN 9575 76), vereda El Salitre, finca Brisas del Llano $5^{\circ} 9,37^{\prime} \mathrm{N}$, 72³4,5’ O (MUJ 4534-35, 4686-88, 5138-41, 5158, 7236), vereda La Playa, finca de Antonio Munévar (MLS 1799-02), vereda Manoguía, finca Veracruz $5^{\circ} 10,082^{\prime} \mathrm{N}, 72^{\circ} 38,190^{\prime} \mathrm{O}$ 
(ICN-TAS 817-18); Nunchía (IAvH 7761); Pore (ICN-RPB 201); Yopal, Aguazul, caño Palestina (MLS 1062).

\section{STRABOMANTIDAE}

Pristimantis medemi* AguAZul, vereda Cunama, finca la Alborada 5¹9'0,12” N, 72³4'59,95” O (MLS 1872, 1889), vereda Cupiagua, quebrada Aguaimara (ICN-TAS 855-58), vereda Cupiagua, quebrada Aguaimara sobre la carretera de 1 vía Pajarito 5¹0,082’ N, 72³8,190’ O (ICNTAS 838-45), vereda Cupiagua, quebrada Hoyo Caliente 5॰14,026’ N, 7240,298' O (ICN-TAS 883-89), vereda El Salitre, finca Brisas del Llano $5^{\circ} 9,37^{\prime} \mathrm{N}, 72^{\circ} 34,5^{\prime} \mathrm{O}$ (MUJ 5127-31, 5133-37, 5161), vereda El Triunfo (MLS 192426); Yopal, quebrada Aguazul (MLS 1061). Pristimantis carranguerorum $^{*}$ AguAZul, vereda Cupiagua, quebrada Hoyo Caliente $5^{\circ} 14,026^{\prime} \mathrm{N}, 7^{\circ} 40,298^{\prime}$ O (ICN-TAS 894). 
Anexo 2. Lista de especies de reptiles con registros para el departamento de Casanare, Colombia

\section{ALLIGATORIDAE}

Caiman crocodilus SAN LuIS de PALENQUe, vereda Guaracura, Colegio Emaús 5’8’40,4” N, 7058’22,6 O (ICN-TAS 230).

\section{AMPHISBAENIDAE}

Amphisbaenaalba NunchÍA, 380 m. (IAvH 5173); Amphisbaena fuliginosa: PAZ DE ARIPORO, vereda La Colombina, entre finca El Porvenir y El Baile (ICN-TAS 813); Amphisbaena sp. PAZ DE ARIPORO, vereda La Colombina, finca El Porvenir 6²’36,5” N, 71'34,2” O (ICN-TAS 700).

\section{ANOMALEPIDIDAE}

Liotyphlops albirostris AGUAZUL, vereda Salitre, finca Brisas del Llano, 510'29” N, 72³3'10” O (MUJ 814).

BOIDAE

Boa constrictor NunCHÍA, ca. $6 \mathrm{~km}$ SE del casco urbano (ICN-JDL 30192); Corallus hortulanus AGUAZUL, vereda Manoguía, finca Veracruz, $5^{\circ} 10,082^{\prime} \mathrm{N}, 72^{\circ} 38,190^{\prime} \mathrm{O}$, 549 m. (ICN-TAS 822); SAN Luis DE PALENQue, vereda Guaracura, Colegio Emaús, 58'40,4” N, 7058'22,6” O; TRINIDAD, vereda San Vicente, finca Candalayes $5^{\circ} 18^{\prime} 47,2^{\prime \prime}$ N, 71²2'5,4" O (ICN-TAS 283, ICN-TAS 600); Epicrates cenchria OROCUÉ, vereda Macucucana, hato El Yopal, Casa del Río (MUJ 823); Villanueva, Palmeras del Oriente (ICNJDL 30177); Eunectesmurinus VILlanueva, Palmeras del Oriente (ICN-JDL 30176).

\section{COLUBRIDAE}

Atractusunivitatus LA SALINA(MLS 290, 2706-7); VILlANUEVA, vereda El Encanto, finca Río Grande (ICN-JDL 30165-6, 30206); Chironius carinatus AgUAZUL, vereda Salitre, finca Brisas del Llano, 510'29”, 72³3'10» O(MUJ 753); NunchíA, pozo Llanura I, Ecopetrol (IAvH 3973); SAN Luis de Palendue, vereda Guaracura, colegio Emaús, 5'8'40,4" N, 7058'22,6" O (ICN-TAS 210); vereda Ulere, finca Altamira $5^{\circ} 11^{\prime} 13,7^{\prime \prime} \mathrm{N}, 70^{\circ} 58^{\prime} 44,2$ " O (ICN-TAS 524, 548); TRINIDAD, vereda La Cañada, finca El Mirador 5'26'18,9" N, 71³6'49" O (ICN-TAS 630-2); finca La Palmita, 5¹9'11,4” N, 71²0'51' O (ICN-TAS 645, 657, $663)$; vereda La Esperanza, finca La Palmita 5²4'15,2” N, 71³7'3,8” O (ICN-TAS 646); Villanueva, Palmeras del Oriente (ICN-JDL 30175); Dipsas pavonia AguAZul, vereda Cupiagua, 650 m. (MLS 359); Dipsas sp. La Salina (MLS 359); Drymarchon sp. TRINIDAD, vereda La Cañada, finca La Palmita, 5¹9'11,4" N, 71²0'51'O (ICN-TAS 643); vereda San Vicente, finca Candalayes, $5^{\circ} 18^{\prime} 47,2^{\prime \prime} \mathrm{N}, 71^{\circ} 22^{\prime} 5,4$ ” O (ICN-TAS 332); Drymoluber rhombifer AGUAZUL, vereda Cunamá, 750 m. (MLS 2981); Erytrholamprus aesculapii Orocué, sobre río Meta (ICN 7057); Helicops angulatus PAZ
DE ARIPORO, finca Jorgín, (ICN 11529); Helicops pastazae YopAL, El Morro, quebrada La Vegana (ICN 10744); Hydrops triangularis SAN LUIS DE PALENQUE, vereda Ulere, finca Altamira, 5'11'13,7' N, 7058'44,2” O (ICN-TAS 235); Imantodes cenchoa AGUAZUL, vereda Cunamá, 750 m. (MLS 2984); Leptodeira annulata AguAZuL, vereda Salitre, finca Brisas del Llano, 5¹0'29” 72॰33'10” O (MUJ 677, 772-4); PAZ DE ARIPORO, finca Jorgín, (ICN 11520-1); TRINIDAD, vereda San Vicente, finca Candalayes $5^{\circ} 18^{\prime} 47,2^{\prime \prime} \mathrm{N}, 71^{\circ} 22^{\prime} 5,4^{\prime \prime} \mathrm{O}$ (ICN-TAS 245, 284); Leptophis ahaetulla Morichal, $350 \mathrm{~m}$. (IAvH 5183), TRINIDAD, vereda La Cañada, finca La Palmita, 5०19'11,4" N, 71²0'51" O (ICN-TAS 602); Ligophis lineatus: Mochuelo (ICN 11200); MANí, (ICN 2835); TRINIDAD, vereda La Cañada, finca El Mirador, 5²6'18,9' $\mathrm{N}$, 71³6'49” O (ICN-TAS 348); Liophis epinephelus LA SALINA, vereda El Arenal (ICN 2854); Liophis melanotus TRINIDAD, vereda La Cañada, finca San Miguel (ICN-TAS 392); vereda San Vicente, finca Candalayes $5^{\circ} 18^{\prime} 47,2^{\prime \prime} \mathrm{N}, 71^{\circ} 22^{\prime} 5,4$ ' O (ICN-TAS 338-9); Liophis reginae LA SALINA (MLS 432); Mastigodryas puchriceps NUNCHÍA, vereda Piedecuesta, finca Las Canarias, 450 m. (IAvH 4767); Mastigodryas sp. MANí, hato La Floresta, Cusiana (ICN 2087); Ninia atrata Aguazul, vereda Salitre, finca Brisas del Llano (MUJ 813) Oxybelis aeneus TRINIDAD, vereda La Cañada, finca La Palmita, 5०19'11,4" N, 71²0’51”O (ICN-TAS 647-9); Oxyrhopus petolarius AGUAZUL, veredaManoguía, finca Veracruz, 5¹0,082’ N, 72³8,190’ O, 549 m. (ICN-TAS 819); PAZ DE ArIPORO, vereda La Colombina, entre finca El Porvenir y El Baile (ICN-TAS 782); TRINIDAD, vereda La Cañada, finca La Palmita, 5¹9'11,4” N, 71²0'51" O (ICNTAS 662); Pseudoboa newiedii Morichal, $350 \mathrm{~m}$. (IAvH 5182); PAZ de Ariporo, finca Jorgín, 5'49'18.8" N, 71'51,26" O (ICN 11527); TRINIDAD, vereda La Cañada, finca La Palmita, 5¹9'11,4" N, 71²0'51" O (ICN TAS 605); Sibon nebulatus Aguazul, vereda Manoguía, finca Veracruz, 549 m. (ICN-TAS 891); Tantilla melanocephala YOPAL, $350 \mathrm{~m}$. (IAvH 5177); Thamnodynastes dixoni (ICN 213); TRINIDAD, vereda La Cañada, finca La Palmita, 5¹9'11,4” N, 71 20 '51' O (ICN-TAS 390, 650-1).

\section{DACTYLOIDAE}

Anolis auratus AgUAzul, vereda Cupiagua, quebrada Aguaimara (ICN-TAS 846); SAN LUIS DE PALENQUe, vereda Guaracura, Colegio Emaús, 58'40,4” N, 7058’22,6” O (ICNTAS 525); Anolis sp.1 Monterrey, caño Lanura (ICN 3988); Anolis sp.2 VillanueVA, casco urbano (ICN-JDL 30135; 30159).

\section{ELAPIDAE}

Micrurus filiformis AguAzul, finca Patio (ICN 11380); Micrurus lemniscatus PAZ DE ARIPORO, finca Jorgín, 549'18.8" N, 71'51'26" N, 7151'26» O (ICN 11526); Micrurus mipartitus pozo Floreña (IAvH 5178) 


\section{GEKKONIDAE}

Hemidactylus angulatus AguAzul, vereda Manoguía, finca Veracruz, 549 m. (ICN-TAS 820); NunCHÍA, vereda Sirivania (ICN-JDL 30200-2); HemIdactylus frenatus PAZ DE ARIPORO, vereda El Totumo, caserío El Totumo (MUJ 1316); SAN LuIS de Palenque, casco urbano (ICN-JDL 30193-8); Trinidad, Hotel El Porvenir, casco urbano (MUJ 1322-6); VILLANUEVA, casco urbano (ICN-JDL 30109-1, 30154, 30163, 302045); Palmeras del Oriente, (ICN-JDL 30190); Hemidactylus palaichtus SAN LUIS DE PALENQUE, vereda Guaracura, Colegio Emaús 58’40,4” N, 7058’22,6” O (ICN-TAS 163, $168,432,435-6,449-55,492-4)$.

\section{GYMNOPHTHALMIDAE}

Gymnophthalmus speciosus AgUAZUL, vereda Salitre, finca Brisas del Llano, 5'10'29”, 72³3'10" O (MUJ 7779); PAZ de ArIPoro, vereda La Colombina, entre finca El Porvenir y El Baile (ICN-TAS 184); San Luis de Palenque, veredaGuaracura, Colegio Emaús 5 ${ }^{\circ}$ '40,4" N, 7058'22,6" O (ICN-TAS 230); Ptychoglossus brevifrontalis Aguazul, vereda Salitre, finca Brisas del Llano, 5०10'29” N, 72³3'10" O (MUJ 775-6).

\section{IGUANIDAE}

Iguana iguana Morichal, 350 m. (IAvH 5180-1); AgUAZUL, vereda Salitre, finca Brisas del Llano, 5 ${ }^{\circ} 10^{\prime} 29^{\prime \prime} \mathrm{N}$, 72³3'10" O (MUJ 759-60); Orocué, vereda Macucucana, hato El Yopal, Casa del Río (MUJ 833); TRINIDAD, vereda La Cañada, finca El Mirador 5²6’18,9” N, 71³6’49” O (ICNTAS 633).

\section{LEPTOTYPHLOPIDAE}

Leptotyphlops jhosuai TAURAMENA (MLS 2482); Leptotyphlops macrolepis AguAZuL, vereda Salitre, finca Brisas del Llano, 510'29” N, 72³3'10” O (MUJ 815).

\section{PODOCNEMIDIDAE}

Podocnemis vogli TRINIDAD, vereda La Cañada, finca La Palmita, 5¹9'11,4” N, 71²0'51”O (ICN-TAS 604).

\section{POLYCHROTIDAE}

Polychrus marmoratus OROCUÉ, vereda Macucucana, hato El Yopal, Casa del Río (MUJ 832).
Sphaerodactylidae

Gonatodes concinnatus AgUAZUL, vereda Cupiagua, quebrada Aguaimara (ICN-TAS ICN-TAS 847); vereda Salitre, finca Brisas del Llano, $5^{\circ} 10^{\prime} 29$ » N, 72 $33^{\prime} 10^{\prime \prime} \mathrm{O}$ (MUJ 75658, 765-68, 812); NunchíA, vereda Piedecuesta, finca Las Canarias, 450 m. (IAvH 4759-60, 4764-6); PAZ De ArIPORO, vereda La Colombina, entre finca El Porvenir y El Baile (ICNTAS 785); vereda La Colombina, finca El Porvenir 6²'36,5” N, 715'34,2” O (ICN-TAS 747-51), San Luis de Palenque, vereda Guaracura, Colegio Emaús $5^{\circ} 8^{\prime} 40,4^{\prime \prime} \mathrm{N}, 70^{\circ} 58^{\prime} 22,6^{\prime \prime}$ O (ICN-TAS 164, 166-67, 234, 434, 526, 528), SAN LUIS DE PALENQUe, vereda Palestina. finca La Lucha $5^{\circ} 23^{\prime} 15,0^{\prime \prime} \mathrm{N}$, 71³7'16,9" O (ICN-TAS 413-5, 424-5); TRINIDAD, vereda La Cañada, finca El Mirador 5²6’18,9” N, 71 $36^{\circ} 49^{\prime \prime}$ O (ICNTAS 347); finca San Miguel (ICN-TAS 410-1); vereda San Vicente, finca Candalayes $5^{\circ} 18^{\prime} 47,2^{\prime \prime} \mathrm{N}, 71^{\circ} 22^{\prime} 5,4^{\prime \prime} \mathrm{O}$ (ICNTAS 243, 316-7, 333-7,340, 346); Villanueva, casco urbano (ICN-JDL 30144-49, 30191); Palmeras de Oriente (301678); Lepidoblepharis sp. Aguazul, vereda Manoguía, finca Veracruz 549 m. (ICN-TAS 866).

\section{TEIIDAE}

Ameiva ameiva Monterrey, $24 \mathrm{~km}$ al suroeste de Monterey (ICN 4738); NunCHíA, vereda Sirivania (ICN-JDL 30199); San Luis De Palenque, vereda Guaracura, Colegio Emaús 58'40,4” N, 7058'22,6 O (ICN-TAS 433, 495), Cnemidophorus gramivagus MONTERREY, $7 \mathrm{~km}$ al noroeste de Monterey (ICN 5231); $8 \mathrm{~km}$ al noroeste de Monterey (ICN 5232); $9 \mathrm{~km}$ al noroeste de Monterey (ICN 5233); 10 $\mathrm{km}$ al noroeste de Monterey (ICN 5234); $11 \mathrm{~km}$ al noroeste de Monterey (ICN 5235); $12 \mathrm{~km}$ al noroeste de Monterey (ICN 5236); $13 \mathrm{~km}$ al noroeste de Monterey (ICN 5237); caño Lanuya (ICN 5248-53); SAN Luis de PALenque, 24 km al suroeste de Monterey (ICN 5225-6); TRINIDAD, vereda San Vicente, finca Candalayes 5 $5^{\circ} 18^{\prime} 47,2^{\prime \prime} \mathrm{N}, 71^{\circ} 22^{\prime} 5,4^{\prime \prime} \mathrm{O}$ (ICNTAS 244); Tupinambis teguixin SAN LUIS de PALENQUe, vereda Guaracura, Colegio Emaús 5 ${ }^{\circ}$ '40,4” N, 7058’22,6” O (ICN-TAS 522); Matamoriche 5 ${ }^{\circ} 9^{\prime} 7,6{ }^{\prime \prime} \mathrm{N}, 70^{\circ} 58^{\prime} 5,4^{\prime \prime} \mathrm{O}$.

\section{VIPERIDAE}

Bothrops atrox TRINIDAD, vereda San Vicente, finca Candalayes $5^{\circ} 18^{\prime} 47,2^{\prime \prime} \mathrm{N}, 71^{\circ} 22^{\prime} 5,4^{\prime \prime}$ O (ICN-TAS 285-6). 